\title{
BREEDING BIRD POPULATIONS AND HABITAT ASSOCIATIONS \\ WITHIN THE SAVANNAH RIVER SITE (SRS)
}

\author{
A COOPERATIVE AGREEMENT \\ between the \\ USDA FOREST SERVICE - SAVANNAH RIVER \\ and the \\ DEPARTMENT OF BIOLOGICAL SCIENCES \\ CLEMSON UNIVERSITY
}

FINAL REPORT

Sideny A. Gauthreaux, Jr. and Steven J. Wagner

Department of Biological Sciences

Clemson University

Clemson, South Carolina 29634-0314 


\section{Introduction}

During the 1970's and 1980's a dramatic decline occurred in the populations of Neotropical migratory birds, species that breed in North America and winter south of the border in Central and South America and in the Caribbean (Wilcove 1988, Treborgh 1989, Robbins et al. 1989, Hagan and Johnston 1992, Finch and Stangel 1993, Peterjohn and Sauer 1994). The debate on the causes of the decline has been intense (Faaborg 2002). Proposed causes range from deforestation in the tropics to increased cowbird parasitism on the breeding grounds because of forest fragmentation (Brittingham and Temple 1983, Faaborg and Arendt 1989, Hutto 1988, Morton and Greenberg 1989). Considerable research is underway or planned to determine the cause(s).

In 1991 an international initiative was mounted by U. S. governmental land management agencies, nongovernmental conservation agencies, and the academic and lay ornithological communities to understand the decline of Neotropical migratory birds in the Americas. The initiative is referred to as the Neotropical Migratory Bird Conservation Program (NMBCP) and is officially called Partners in Flight because of its international mission. For the last several years ornithologists and land managers have engaged in formal discussions about programs that would 1) inventory Neotropical migratory birds in various habitats, 2) begin studies that would measure the impact of various land management practices on Neotropical migratory bird populations, and 3) initiate a long-term monitoring program that would provide managers with information on the effectiveness of their practices to conserve Neotropical migrants. 
In cooperation with the USDA Forest Service - Savannah River (FS - SR) we began 1992 a project directed to monitoring population densities of breeding birds using the Breeding Bird Census (BBC) methodology in selected habitats within the Savannah River Site SRS. In addition we related point count data on the occurrence of breeding Neotropical migrants and other bird species to the habitat data gathered by the Forest Inventory and Analysis (FIA) program of the USDA Forest Service (McClure et al. 1979, Sheffield 1981), and data on habitat treatments within forest stands.

Ultimately the monitoring data can be used to measure the impact of various forest management practices on the biodiversity of bird species in different landscapes not only within SRS, but throughout the state of South Carolina and the entire Southeast.

Kilgo et al. (2000) used data from the Breeding Bird Survey (BBS) and point count data from several studies (including ours) on SRS to compare breeding bird populations on and off the SRS property. They found that the SRS avifauna differed from the regional avifauna. Species that prefer forested habitats were more abundant and species that prefer agricultural and urban habitats were less abundant on SRS than in the surrounding region. These results are consistent with the differences between habitats on SRS and the surrounding area. When the site was established in 1951 a reforestation program began and forested land has increased from 48\% to $89 \%$ of the total land cover on the site (White and Gaines 2000, Kilgo et al. 2000). The surrounding upper coastal plain of South Carolina consists of only 62\% forested habitats (Kilgo et al. 2000).

In this report we summarize 10 years of Breeding Bird Census data collected from eight plots on SRS. Population trends on seven of these plots are compared with trend data from the Breeding Bird Survey and from two other study plots (mixed pine deciduous and bottomland) 
located just south of the Savannah River Plant near Martin, SC. We also report on changes in bird numbers and species composition on another plot (clear-cut -longleaf pine regeneration) that has experienced successional vegetation changes.

\section{Methods}

\section{Breeding Bird Censuses}

The breeding bird census methodology follows Ralph et al. (1993). The vegetation classification on the breeding bird census plots follows the classification outlined in the

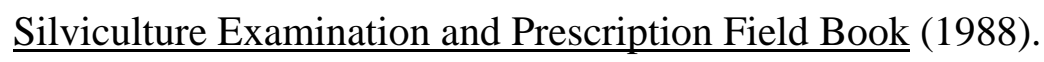

During the breeding season of 1992, six Breeding Bird Census (BBC) plots were established and censused within the SRS. Each forested plot measured approximately 12.5 ha. Another plot of approximately 25 ha was established in a clear-cut/longleaf pine regeneration stand. The five forested plots included areas of mature longleaf pine, mature loblolly pine (Loblolly I), mature upland hardwood, mature bottomland hardwood, and mature swamp forest. Detailed plot descriptions are provided in the Journal of Field Ornithology, Vol. 64 Supplement and Appendix I. During the breeding season of 1993, two additional forested plots were established within the SRS, one in a mature loblolly pine stand (Loblolly II), the other in a stand of scrub oak with scattered longleaf pine. Detailed descriptions of these plots are provided in the Journal of Field Ornithology, Vol. 65 Supplement and Appendix I. In 1995 a new 11-20 year old loblolly pine plot was established to replace the original mature loblolly plot (Loblolly I). The original plot was abandoned after 1992 because of alteration of the surrounding landscape. The new plot is described in the Journal of Field Ornithology, Vol. 67 Supplement and Appendix I. 
For each year through 1995, BBC results from the SRS plots were published in the Journal of Field Ornithology Supplements. Publication of the Supplements was discontinued after 1995. Table 1 summarizes the census history for the BBC plots. In 1993 four Breeding Bird Censuses were conducted in the following habitat types: longleaf pine, loblolly pine, scrub oak/longleaf pine, and clear-cut/longleaf pine regeneration (Journal of Field Ornithology, Vol. 65 Supplement: Pp. 75, 77-78, 94, 125-126). During the breeding season of 1994, four Breeding Bird Censuses were conducted in the following habitat types: upland hardwood, mature loblolly pine, bottomland hardwood, and clear-cut/longleaf pine regeneration ( Ornithology, Vol. 66 Supplement: Pp. 61-63, 72, 116). The 1995 season included censuses in swamp forest, scrub oak, 11-20 year old loblolly pine, and clear-cut/longleaf pine regeneration (Journal of Field Ornithology, Vol. 67 Supplement: Pp. 58, 67-68, 89). Because of the rapid changes in the vegetation of the clear-cut/longleaf pine regeneration plot, the area that was censused in 1995 was reduced from the original 25 ha to 12.5 ha so that it could be covered thoroughly. The height and density of vegetation made a larger area difficult to census in the amount of time allotted.

The 1996 season included censuses in upland hardwood, longleaf pine, bottomland hardwood, and clear-cut/longleaf pine regeneration plots. The 1997 season included censuses in the 11-20 year old loblolly, mature loblolly, scrub oak, and clear-cut/longleaf pine regeneration plots. The 1998 season included censuses in swamp forest, upland hardwood, longleaf pine, and clear-cut/longleaf pine regeneration plots. In 1999, the bottomland hardwood, scrub oak, 11-20 year old loblolly, and clear-cut/longleaf pine regeneration plots were censused. The clearcut/longleaf pine regeneration plot was the only plot censused in 2000 and 2001. 
With the exception of the clear-cut/longleaf pine regeneration plot, we followed a schedule with two- to three-year intervals between censuses on each plot (Table 1). The clearcut/longleaf pine regeneration plot was censused for 10 consecutive years while each of the other plots was censused only 3-4 times during those 10 years.

Table 1. History of Breeding Bird Censuses conducted on plots located on SRS

\begin{tabular}{|c|c|c|c|c|c|c|c|c|}
\hline & \multicolumn{8}{|c|}{ Breeding Bird Census Plots } \\
\hline & Upland & $\begin{array}{l}\text { Clear-cut } \\
\text { Regen. }\end{array}$ & Swamp & $\begin{array}{c}\text { Longleaf } \\
\text { Pine }\end{array}$ & $\begin{array}{l}\text { Loblolly } \\
\text { Pine I(a) }\end{array}$ & Bottomland & $\begin{array}{l}\text { Loblolly } \\
\text { Pine II }\end{array}$ & Scrub Oak \\
\hline Year & & & & & & & & \\
\hline 1992 & $\mathbf{X}$ & $\mathbf{X}$ & $\mathbf{X}$ & $\mathbf{X}$ & $\mathbf{X}$ & $\mathbf{X}$ & & \\
\hline 1993 & & $\mathbf{X}$ & & $\mathbf{X}$ & & & $\mathbf{X}$ & $\mathbf{X}$ \\
\hline 1994 & $\mathbf{X}$ & $\mathbf{X}$ & & & & $\mathbf{X}$ & $\mathbf{X}$ & \\
\hline 1995 & & $\mathbf{X}$ & $\mathbf{X}$ & & $\mathbf{X}$ & & & $\mathbf{X}$ \\
\hline 1996 & $\mathbf{X}$ & $\mathbf{X}$ & & $\mathbf{X}$ & & $\mathbf{X}$ & & \\
\hline 1997 & & $\mathbf{X}$ & & & $\mathbf{X}$ & & $\mathbf{X}$ & $\mathbf{X}$ \\
\hline 1998 & $\mathbf{X}$ & $\mathbf{X}$ & $\mathbf{X}$ & $\mathbf{X}$ & & & & \\
\hline 1999 & & $\mathbf{X}$ & & & $\mathbf{X}$ & $\mathbf{X}$ & & $\mathbf{X}$ \\
\hline 2000 & & $\mathbf{X}$ & & & & & & \\
\hline 2001 & & $\mathbf{X}$ & & & & & & \\
\hline
\end{tabular}

a - The original 40 year old plot was abandoned after 1992 because of alteration of the surrounding landscape. This plot was replaced in 1995 with a loblolly plot in the 11-20 year old condition class (see Appendix I). 


\section{Clariant Corporation (Sandoz, Inc.) Plots}

From 1978 through 1993, S. A. Gauthreaux conducted limited area bird censuses at 22 sites (each 3 hectare) on property owned by the Clariant Corporation (formerly Sandoz, Inc.) south of the Savannah River Site and the settlement of Martin in Allendale County. The censuses of breeding birds were always conducted in late May and the time in a plot was always 45 minutes. The purpose of these censuses was to evaluate the environmental impact of a living filtration system (where industrial waste water was sprayed in pine forests) on bird populations. For the comparative analysis in this report, two control plots (Spray Control A and Lagoon Control A) were combined into a mixed pine/deciduous plot. The plots contained mixed woodland (approximately 50\% pine and 50\% deciduous), and the deciduous trees were mostly oaks (Quercus) and 30-50 feet in height. The soil was sandy and understory vegetation of shrubs and grasses occupied $60 \%$ of the ground area in the plot. The floodplain plot we discuss in this report is actually made up of four of the original Sandoz plots--Spray Drainage A, Spray Drainage B, Spray Drainage C, and Lagoon Drainage A. All of these plots were in the floodplain of the Savannah River and contained bottomland forest (tupelo, blackgum, sweetgum, oaks, and some cypress). The plots on the edge of the river also contained some cottonwood, willow, ash, elm, hackberry, and maple. The understory was generally thick and contained vines, shrubs, and cane and on a few occasions the plots close to the river were flooded or contained standing water. 


\section{Analyses}

We compare the BBC data with census data from two other sources, the Breeding Bird Survey (BBS) and two nearby census plots (mixed pine deciduous and bottomland) located on property managed by Clariant Corporation (formerly Sandoz, Inc.) in Allendale county.

The raw data (territories/40 ha) for each of the forested BBC plots are presented in Appendix II. The data for the Clear-cut/Longleaf Pine Regeneration plot are presented in Table 4. Population trends on the BBC plots were examined using linear regression (Tables 2-4). We used the route-regression method for estimation of population trends in the BBS data (Sauer 2003). We performed two sets of analyses using BBS data, one for USFWS Region 4 (southeastern U. S.) and the other for the physiographic region Upper Coastal Plain. Both analyses cover the time period of 1978-2002 which incorporates the years that censuses were conducted on the Clariant (Sandoz) property and on our BBC plots at SRS.

\section{Results and Discussion}

\section{Forested BBC Plots}

Results of the BBS, Clariant (Sandoz), and BBC population trend analyses are presented in Table 2 for permanent resident species and in Table 3 for migrant species. Among permanent resident species, only two species showed significant changes on the forested BBC plots.

Carolina Chickadees declined on the Bottomland Hardwood plot and Pine Warblers declined on the Longleaf plot. Carolina Chickadees also showed significant declines in both BBS analyses and on the Floodplain census plot at Clariant (Sandoz). Pine Warblers showed significant 
increases in both BBS analyses and a significant decline in the Floodplain plot at Clariant (Sandoz).

Among migrant species, Yellow-billed Cuckoos showed a significant increase on the BBC Bottomland Hardwood plot. Both regional BBS analyses reported significant declines for this species. On the Upland Hardwood plot, Wood Thrush, Hooded Warbler, and Summer Tanager all had significant declines. Summer Tanagers also declined significantly on the Loblolly II plot. There were also significant declines for Wood Thrushes in both sets of BBS analyses and in the Clariant (Sandoz) Floodplain plot. Hooded Warblers had significant declines in both of the Clariant (Sandoz) plots but not in the BBS analyses. There were no significant changes in Summer Tanager numbers in the BBS data or on the Clariant (Sandoz) plots.

Only six species had significant changes in numbers on the SRS BBC plots. The BBS data set had 31 species with significant changes in the southeastern region analysis and 20 species with significant changes in the upper coastal plain analysis (Tables 2 and 3). Twelve of the species with significant changes in the BBS data did not occur on the BBC plots frequently enough to allow analysis (e.g. Northern Bobwhite, Northern Mockingbird, Common Grackle, and Painted Bunting). Even if these twelve species are excluded from consideration, there appears to be a difference between the numbers of species showing significant population trends in the regional data when compared to the SRS BBC data. The results indicate that populations are more stable on the SRS BBC plots. However, the BBC data may not be adequate to detect changes in bird populations. The forested BBC plots have been censused only 3 or 4 times during the study period (Table 1). The ability of statistical tests, such as linear regression, to detect significant population tends is probably limited by this small number of censuses. Another potentially confounding factor is the number of different observers who have conducted 
these censuses. Observer effects have been cited as a confounding variable in many studies involving census data (Sauer et al. 1994). 
Table 2. Population trend analyses for permanent resident species on BBS routes, Sandoz property, and BBC plots at SRS.

\begin{tabular}{|c|c|c|c|c|c|c|c|c|}
\hline \multirow[b]{3}{*}{ Species } & \multicolumn{4}{|c|}{ BBS (1978-2002) } & \multicolumn{4}{|c|}{ Sandoz (1978-1993) } \\
\hline & \multicolumn{2}{|c|}{ Southeast } & \multicolumn{2}{|c|}{ Upper Coastal Plain } & \multicolumn{2}{|c|}{ Floodplain } & \multicolumn{2}{|c|}{ Mixed } \\
\hline & trend & $p$ value & trend & $p$ value & trend & $p$ value & trend & $p$ value \\
\hline \multicolumn{9}{|l|}{ Northern Bobwhite } \\
\hline Mourning Dove & -0.63 & 0.02017 & -1.87 & 0.00000 & 0.06 & 0.7494 & -0.38 & 0.0494 \\
\hline \multicolumn{9}{|l|}{ Common Ground-Dove } \\
\hline \multicolumn{9}{|l|}{ Red-headed woodpecker } \\
\hline Red-bellied Woodpecker & 0.25 & 0.22058 & -0.16 & 0.55224 & 0.17 & 0.3996 & -0.1 & 0.6259 \\
\hline Downy Woodpecker & -0.46 & 0.22138 & -1.24 & 0.04100 & -0.1 & 0.6074 & -0.15 & 0.459 \\
\hline Blue Jay & -1.33 & 0.00000 & -1.70 & 0.00000 & -0.32 & 0.0987 & -0.44 & 0.0245 \\
\hline American Crow & 1.48 & 0.00000 & 1.63 & 0.00000 & -0.06 & 0.7491 & -0.2 & 0.3068 \\
\hline Fish Crow & 0.44 & 0.53363 & 0.42 & 0.58394 & -0.06 & 0.7426 & -0.62 & 0.0012 \\
\hline Carolina Chickadee & -1.28 & 0.00008 & -2.12 & 0.00001 & -0.4 & 0.0359 & -0.15 & 0.4376 \\
\hline Tufted Titmouse & 1.04 & 0.00001 & 0.74 & 0.01738 & -0.34 & 0.0805 & 0.13 & 0.4904 \\
\hline White-breated Nuthatch & 1.94 & 0.00288 & 3.30 & 0.16308 & & & & \\
\hline Brown-headed Nuthatch & -0.83 & 0.34550 & -2.24 & 0.12402 & -0.17 & 0.4229 & -0.38 & 0.049 \\
\hline Carolina Wren & 0.85 & 0.00010 & 0.63 & 0.04593 & -0.54 & 0.0047 & -0.27 & 0.1628 \\
\hline Eastern Bluebird & 3.30 & 0.00000 & 3.33 & 0.00000 & 0.04 & 0.8676 & 0.12 & 0.548 \\
\hline \multicolumn{9}{|l|}{ Northern Mockingbird } \\
\hline Brown Thrasher & -0.15 & 0.57637 & -0.08 & 0.85479 & & & & \\
\hline Pine Warbler & 0.67 & 0.04805 & 1.59 & 0.00389 & -0.56 & 0.0041 & -0.07 & 0.74 \\
\hline Eastern Towhee & -1.14 & 0.00000 & -0.65 & 0.06397 & -0.25 & 0.2112 & -0.31 & 0.1011 \\
\hline Bachman's Sparrow & -0.53 & 0.84528 & 5.67 & 0.57624 & -0.31 & 0.1585 & -0.07 & 0.7313 \\
\hline Chipping Sparrow & 1.71 & 0.00020 & 1.00 & 0.06077 & & & & \\
\hline Field Sparrow & -1.49 & 0.00004 & -1.25 & 0.05179 & & & & \\
\hline Northern Cardinal & 0.11 & 0.45424 & -0.14 & 0.50796 & -0.39 & 0.0433 & -0.52 & 0.0075 \\
\hline Eastern Meadowlark & -3.86 & 0.00000 & -4.82 & 0.00000 & & & & \\
\hline Common Grackle & -2.67 & 0.00000 & -2.22 & 0.00000 & & & & \\
\hline Brown-headed Cowbird & -1.38 & 0.00001 & -1.87 & 0.00002 & 0.06 & 0.7437 & 0.06 & 0.7469 \\
\hline
\end{tabular}


Table 2. (cont.)

\begin{tabular}{|c|c|c|c|c|c|c|c|c|c|c|c|c|c|c|}
\hline \multirow[b]{3}{*}{ Species } & \multirow{2}{*}{\multicolumn{2}{|c|}{$\begin{array}{c}\text { Bottomland } \\
\text { Hardwood }\end{array}$}} & \multicolumn{10}{|c|}{ Savannah River Site (1992-2001) } & \multirow{2}{*}{\multicolumn{2}{|c|}{ Loblolly I }} \\
\hline & & & & amp & & $\begin{array}{l}\text { land } \\
\text { wood }\end{array}$ & $\mathrm{Scl}$ & Oak & & gleaf & & olly II & & \\
\hline & trend & $p$ value & trend & $p$ value & trend & $p$ value & trend & $p$ value & trend & $p$ value & trend & $p$ value & trend & $p$ value \\
\hline \multicolumn{15}{|l|}{ Northern Bobwhite } \\
\hline Mourning Dove & & & & & & & -0.66 & 0.3675 & & & & & & \\
\hline $\begin{array}{l}\text { Common Ground-Dove } \\
\text { Red-headed } \\
\text { woodpecker } \\
\text { Red-bellied } \\
\text { Woodpecker }\end{array}$ & -0.88 & 0.2789 & -0.3 & 0.3333 & -0.49 & 0.5955 & -0.66 & 0.1056 & 0.08 & 0.8316 & -0.12 & 0.8211 & & \\
\hline Downy Woodpecker & -0.84 & 0.1908 & -0.61 & 0.3333 & 0.08 & 0.8607 & & & 0.39 & 0.3342 & & & & \\
\hline $\begin{array}{l}\text { Blue Jay } \\
\text { American Crow } \\
\text { Fish Crow }\end{array}$ & & & & & & & -1.55 & 0.0561 & & & & & & \\
\hline Carolina Chickadee & -0.85 & 0.042 & -1.21 & 0.1719 & -1.22 & 0.2971 & 0.1952 & 0.66 & -0.13 & 0.7238 & 0.76 & 0.1678 & 0 & 1 \\
\hline $\begin{array}{l}\text { Tufted Titmouse } \\
\text { White-breated } \\
\text { Nuthatch } \\
\text { Brown-headed } \\
\text { Nuthatch }\end{array}$ & -0.03 & 0.9615 & $\begin{array}{l}0.9 \\
-1.2\end{array}$ & $\begin{array}{l}0.121 \\
0.1789\end{array}$ & $\begin{array}{l}0.56 \\
-0.07\end{array}$ & $\begin{array}{l}0.4746 \\
0.8771\end{array}$ & 0 & 1 & -0.07 & 0.9383 & -1.01 & 0.3619 & -0.82 & 0.3333 \\
\hline Carolina Wren & -0.43 & 0.575 & -0.3 & 0.9164 & -1.15 & 0.1294 & & & & & 0.26 & 0.9169 & & \\
\hline $\begin{array}{l}\text { Eastern Bluebird } \\
\text { Northern Mockingbird }\end{array}$ & & & & & & & & & -0.55 & 0.2883 & & & & \\
\hline Brown Thrasher & & & & & & & -0.57 & 0.0577 & & & & & & \\
\hline Pine Warbler & & & & & & & -1.87 & 0.2483 & -2.81 & 0.0083 & 0.38 & 0.8211 & -0.4 & 0.3333 \\
\hline Eastern Towhee & & & & & & & & & -0.82 & 0.5852 & 0.51 & 0.7197 & & \\
\hline Bachman's Sparrow & & & & & & & & & -1.89 & 0.0659 & & & & \\
\hline $\begin{array}{l}\text { Chipping Sparrow } \\
\text { Field Sparrow }\end{array}$ & & & & & & & & & 0.08 & 0.9657 & & & & \\
\hline $\begin{array}{l}\text { Northern Cardinal } \\
\text { Eastern Meadowlark } \\
\text { Common Grackle } \\
\text { Brown-headed Cowbird }\end{array}$ & -1.81 & 0.1837 & & & -1.56 & 0.2579 & 0.24 & 0.7476 & & & 0.33 & 0.9341 & -0.8 & 0.3333 \\
\hline
\end{tabular}


Table 3. Population trend analyses for migrant species on BBS routes, Sandoz property, and BBC plots at SRS.

\begin{tabular}{|c|c|c|c|c|c|c|c|c|}
\hline \multirow[b]{3}{*}{ Species } & \multicolumn{4}{|c|}{ BBS (1978-2002) } & \multicolumn{4}{|c|}{ Sandoz (1978-1993) } \\
\hline & \multicolumn{2}{|c|}{ Southeast } & \multicolumn{2}{|c|}{ Upper Coastal Plain } & \multicolumn{2}{|c|}{ Floodplain } & \multicolumn{2}{|c|}{ Mixed } \\
\hline & trend & $\mathrm{p}$ value & trend & $\mathrm{p}$ value & trend & $p$ value & trend & $p$ value \\
\hline $\begin{array}{l}\text { Yellow-billed Cuckoo } \\
\text { Ruby-throated }\end{array}$ & -2.25 & 0.00000 & -3.00 & 0.00000 & -0.31 & 0.1137 & -0.01 & 0.9627 \\
\hline Hummingbird & 0.26 & 0.68723 & -0.75 & 0.44339 & -0.28 & 0.1523 & -0.19 & 0.3645 \\
\hline Eastern Wood-Pewee & -1.45 & 0.00000 & -1.60 & 0.00112 & -0.07 & 0.7508 & 0.08 & 0.6992 \\
\hline Acadian Flycatcher & 0.27 & 0.47739 & 0.82 & 0.15512 & -0.53 & 0.0054 & 0.69 & 0.0004 \\
\hline Great Crested Flycatcher & 1.02 & 0.00014 & 0.48 & 0.30292 & -0.04 & 0.8193 & -0.23 & 0.2439 \\
\hline Eastern Kingbird & -0.50 & 0.14291 & -0.15 & 0.76676 & 0.26 & 0.2328 & -0.24 & 0.246 \\
\hline White-eyed Vireo & 0.33 & 0.13705 & 0.64 & 0.02694 & -0.68 & 0.0003 & 0.15 & 0.4331 \\
\hline Yellow-throated Vireo & 1.60 & 0.01824 & 2.05 & 0.07013 & 0.06 & 0.7469 & 0.08 & 0.717 \\
\hline Red-eyed Vireo & 0.74 & 0.00548 & 0.21 & 0.55127 & 0.08 & 0.6781 & -0.42 & 0.0328 \\
\hline Blue-gray Gnatcatcher & 0.63 & 0.12387 & 0.90 & 0.20767 & -0.21 & 0.2723 & 0.43 & 0.0207 \\
\hline Wood Thrush & -1.09 & 0.00343 & -0.92 & 0.03315 & -0.58 & 0.0027 & 0.25 & 0.2209 \\
\hline Gray Catbird & -2.22 & 0.00016 & -0.49 & 0.53214 & & & & \\
\hline Northern Parula & -0.06 & 0.9015 & 0.43 & 0.53502 & -0.62 & 0.001 & -0.28 & 0.1503 \\
\hline Yellow-throated Warbler & 0.17 & 0.83503 & -2.17 & 0.26077 & 0.08 & 0.6781 & 0.13 & 0.5384 \\
\hline Prairie Warbler & -1.46 & 0.00924 & -0.89 & 0.22860 & 0.03 & 0.8745 & -0.12 & 0.5617 \\
\hline Black-and-white Warbler & -1.70 & 0.01134 & -4.16 & 0.00085 & & & & \\
\hline American Redstart & 3.26 & 0.00927 & 0.26 & 0.87115 & & & & \\
\hline Prothonotary Warbler & -1.39 & 0.15392 & -1.67 & 0.37532 & 0.03 & 0.8901 & & \\
\hline Swainson's Warbler & 1.62 & 0.51245 & 5.25 & 0.21048 & -0.81 & 0.0001 & & \\
\hline Ovenbird & 0.46 & 0.43448 & -0.18 & 0.76254 & & & & \\
\hline Louisiana Waterthrush & 0.41 & 0.64399 & -1.37 & 0.19561 & 0.12 & 0.5575 & & \\
\hline Kentucky Warbler & -1.55 & 0.00116 & -2.39 & 0.00004 & -0.26 & 0.1812 & 0.21 & 0.3408 \\
\hline Common Yellowthroat & -1.31 & 0.00000 & -0.32 & 0.56215 & & & & \\
\hline Hooded Warbler & 0.30 & 0.50462 & 0.89 & 0.09773 & -0.7 & 0.0002 & -0.45 & 0.039 \\
\hline Yellow-breasted Chat & 0.77 & 0.00435 & 1.51 & 0.00003 & 0.4 & 0.0589 & -0.01 & 0.9587 \\
\hline Summer Tanager & -0.11 & 0.79427 & -0.41 & 0.42867 & -0.17 & 0.3996 & 0.02 & 0.9262 \\
\hline Blue Grosbeak & 0.87 & 0.00674 & 0.67 & 0.09422 & 0.13 & 0.5449 & -0.19 & 0.3411 \\
\hline Indigo Bunting & -0.22 & 0.24466 & 0.11 & 0.66801 & 0.61 & 0.0021 & 0.23 & 0.26 \\
\hline Painted Bunting & -3.63 & 0.00031 & -0.41 & 0.76688 & 0.07 & 0.7449 & 0.18 & 0.3661 \\
\hline Orchard Oriole & -0.44 & 0.30799 & 0.84 & 0.21183 & 0.5 & 0.0212 & -0.07 & 0.7313 \\
\hline
\end{tabular}


Table 3. (cont.)

\begin{tabular}{|c|c|c|c|c|c|c|c|c|c|c|c|c|c|c|}
\hline \multirow[b]{3}{*}{ Species } & \multicolumn{14}{|c|}{ Savannah River Site (1992-2001) } \\
\hline & \multicolumn{2}{|c|}{ Bottomland Hardwood } & \multicolumn{2}{|c|}{ Swamp } & \multicolumn{2}{|c|}{ Upland Hardwood } & \multicolumn{2}{|c|}{ Scrub Oak } & \multicolumn{2}{|c|}{ Longleaf } & \multicolumn{2}{|c|}{ Loblolly II } & \multicolumn{2}{|c|}{ Loblolly I } \\
\hline & trend & $\mathrm{p}$ value & trend & $p$ value & trend & $p$ value & trend & $p$ value & trend & $p$ value & trend & $p$ value & trend & $p$ value \\
\hline Yellow-billed Cuckoo & 0.89 & 0.0381 & 0.30 & 0.6766 & 0.17 & 0.5528 & -0.66 & 0.3675 & & & & & & \\
\hline \multicolumn{15}{|l|}{ Ruby-throated Hummingbird } \\
\hline Eastern Wood-Pewee & & & & & & & & & 1.51 & 0.1231 & -1.01 & 0.3619 & & \\
\hline Acadian Flycatcher & 1.43 & 0.0971 & -3.33 & 0.1618 & -0.49 & 0.8471 & & & & & & & & \\
\hline Great Crested Flycatcher & -0.72 & 0.3124 & -0.61 & 0.3333 & & & -1.15 & 0.1597 & -0.7 & 0.3785 & -0.99 & 0.3764 & -0.82 & 0.3333 \\
\hline \multicolumn{15}{|l|}{ Eastern Kingbird } \\
\hline White-eyed Vireo & -2.05 & 0.2899 & & & -0.57 & 0.0577 & & & & & & & & \\
\hline Yellow-throated Vireo & & & & & -0.4 & 0.7128 & & & & & & & & \\
\hline Red-eyed Vireo & -1.06 & 0.5538 & 0.60 & 0.5542 & -0.83 & 0.6833 & & & & & & & & \\
\hline Blue-gray Gnatcatcher & 1.85 & 0.1048 & 0.00 & 1 & 0.49 & 0.4467 & 1.8 & 0.4118 & & & & & & \\
\hline Wood Thrush & -1.26 & 0.2622 & & & -1.71 & 0.0159 & -0.81 & 0.089 & & & & & & \\
\hline \multicolumn{15}{|l|}{ Gray Catbird } \\
\hline Northern Parula & 0.26 & 0.9374 & -1.82 & 0.2349 & 1.14 & 0.8384 & & & & & & & & \\
\hline Yellow-throated Warbler & & & & & 0.57 & 0.8407 & & & & & & & & \\
\hline Prairie Warbler & & & & & & & & & -3.31 & 0.2783 & & & & \\
\hline Black-and-white Warbler & -0.39 & 0.2649 & & & -0.65 & 0.4108 & -0.16 & 0.7418 & & & & & & \\
\hline American Redstart & 0.16 & 0.9543 & & & -0.16 & 0.8652 & & & & & & & & \\
\hline Prothonotary Warbler & & & -1.22 & 0.6151 & & & & & & & & & & \\
\hline Swainson's Warbler & 0.71 & 0.3296 & & & & & & & & & & & & \\
\hline Ovenbird & & & & & & & -0.41 & 0.3318 & & & & & & \\
\hline Louisiana Waterthrush & & & & & 0.165 & 0.8652 & & & & & & & & \\
\hline Kentucky Warbler & -0.56 & 0.7574 & & & -2.71 & 0.0972 & & & & & & & & \\
\hline \multicolumn{15}{|l|}{ Common Yellowthroat } \\
\hline Hooded Warbler & -1.67 & 0.6802 & & & -2.38 & 0.0087 & & & & & & & & \\
\hline \multicolumn{15}{|l|}{ Yellow-breasted Chat } \\
\hline Summer Tanager & -0.21 & 0.7959 & -0.62 & 0.6616 & -0.82 & 0.0001 & -1.15 & 0.1597 & -0.97 & 0.0565 & -2.08 & 0.0366 & & \\
\hline Blue Grosbeak & & & & & & & & & -0.43 & 0.5552 & & & & \\
\hline Indigo Bunting & & & & & & & & & & & 1.95 & 0.1075 & & \\
\hline Painted Bunting & & & & & & & & & & & & & & \\
\hline Orchard Oriole & & & & & & & & & & & & & & \\
\hline
\end{tabular}




\section{Clear-cut/Longleaf pine Regeneration Plot}

The raw data and analysis results for the Clear-cut/Longleaf Pine Regeneration plot are presented in Table 4. As the plot has matured Northern Bobwhite, Common Ground-dove, Redheaded Woodpecker, Red-bellied Woodpecker, Eastern Kingbird, Eastern Bluebird, Northern Mockingbird, Bachman’s Sparrow, and Orchard Oriole have all shown significant declines. None of these species were encountered on the plot after 1998. Two species, White-eyed Vireo and Pine Warbler, have shown significant increases.

The population trends observed on the plot are not surprising given the successional changes taking place as the plot has matured. Most of the species showing significant declines are species typical of more open or edge habitats (Hamel 1992). The two woodpecker species are an exception. However, their decline is primarily the result of the 1995 reduction in the area being censused. This eliminated a small stand ( 0.5 ha) of hardwoods and several snags where these species were typically found. White-eyed Vireos showed a significant increase as understory vegetation matured in several areas of the plot and along its edges. Pine Warblers were first counted in the plot in 1999 when the plot was 9 years old and the Longleaf pines had reached 4-8 $\mathrm{m}$ in height. A nest and fledglings were found on the plot in 2001 when the pines had reached $10 \mathrm{~m}$ in height in some areas and produced a more or less continuous canopy in part of the eastern end of the plot.

There has also been a significant decline in species diversity on the plot (Table 4). The number of species recorded dropped from 30 to 14. Part of this decline may be attributable to the 1995 reduction in the area being censused which reduced some of the heterogeneity of the plot. 
Table 4. Clear-cut-longleaf pine regeneration plot data (territories/40 ha) and analyses.

\begin{tabular}{|c|c|c|c|c|c|c|c|c|c|c|c|c|}
\hline Year & 1992 & 1993 & 1994 & 1995 & 1996 & 1997 & 1998 & 1999 & 2000 & 2001 & & \\
\hline Observer & $\mathrm{DD}$ & SW & $\mathrm{CI}$ & $\mathrm{CI}$ & MG & MG & SW & SW & SW & SW & & \\
\hline Plot area (ha) & 25 & 25 & 25 & 12.5 & 12.5 & 12.5 & 12.5 & 12.5 & 12.5 & 12.5 & & \\
\hline Census Trips & 8 & 8 & 10 & 10 & 10 & 10 & 10 & 10 & 10 & 10 & & \\
\hline Stand Age (years) & 2 & 3 & 4 & 5 & 6 & 7 & 8 & 9 & 10 & 11 & & \\
\hline & & & & & & & & & & & trend & p value \\
\hline Northern Bobwhite & 20 & 4.8 & 9.6 & 9.6 & $\mathbf{0}$ & $\mathbf{0}$ & 8 & $\mathbf{0}$ & $\mathbf{0}$ & $\mathbf{0}$ & -1.61 & 0.016 \\
\hline Mourning Dove & 12.8 & 0 & 11.2 & 12.8 & 0 & 0 & 0 & 3.2 & 3.2 & 3.2 & -0.86 & 0.162 \\
\hline Common Ground-dove & 3.2 & 4 & 4.8 & 3.2 & 6.4 & 3.2 & 4.8 & $\mathbf{0}$ & $\mathbf{0}$ & $\mathbf{0}$ & -0.48 & 0.045 \\
\hline $\begin{array}{l}\text { Yellow-billed Cuckoo } \\
\text { Red-headed }\end{array}$ & 0.8 & 0 & 0 & 0 & 0 & 0 & 0 & 0 & 0 & 0 & & \\
\hline $\begin{array}{l}\text { Woodpecker } \\
\text { Red-bellied }\end{array}$ & 3.2 & 4 & 4.8 & 4.8 & $\mathbf{0}$ & $\mathbf{0}$ & $\mathbf{0}$ & $\mathbf{0}$ & $\mathbf{0}$ & $\mathbf{0}$ & -0.58 & 0.007 \\
\hline Woodpecker & 2.4 & 2.4 & 4.8 & 3.2 & 3.2 & $\mathbf{0}$ & $\mathbf{0}$ & $\mathbf{0}$ & $\mathbf{0}$ & $\mathbf{0}$ & -0.45 & 0.01 \\
\hline Downy Woodpecker & 0 & 0 & 0 & 1.6 & 0 & 0 & 0 & 0 & 0 & 0 & & \\
\hline Northern Flicker & 3.2 & 1.6 & 2.4 & 3.2 & 0 & 0 & 0 & 0 & 0 & 0 & & \\
\hline Great Crested Flycatcher & 3.2 & 2.4 & 4 & 3.2 & 6.4 & 3.2 & 0 & 0 & 3.2 & 0 & -0.34 & 0.142 \\
\hline Eastern Kingbird & 4.8 & 2.4 & 4 & 6.4 & 4.8 & $\mathbf{0}$ & $\mathbf{0}$ & $\mathbf{0}$ & $\mathbf{0}$ & $\mathbf{0}$ & -0.63 & 0.013 \\
\hline White-eyed Vireo & 4.8 & 0.8 & 3.2 & 3.2 & $\mathbf{0}$ & 4.8 & 11.2 & 11.2 & 9.6 & 6.4 & 0.88 & 0.038 \\
\hline Blue Jay & 1.6 & 0 & 0 & 1.6 & 0 & 0 & 0 & 0.8 & 0 & 1.6 & -0.01 & 0.958 \\
\hline Carolina Chickadee & 0 & 0.8 & 0 & 0 & 9.6 & 6.4 & 3.2 & 1.6 & 3.2 & 3.2 & 0.36 & 0.32 \\
\hline Tufted Titmouse & 0.8 & 0 & 0 & 0 & 0 & 0 & 0 & 0 & 3.2 & 0 & & \\
\hline Brown-headed Nuthatch & 0.8 & 0 & 0 & 3.2 & 0 & 0 & 0 & 0 & 0 & 0 & & \\
\hline Carolina Wren & 9.6 & 4.8 & 6.4 & 3.2 & 12.8 & 8 & 6.4 & 1.6 & 1.6 & 4.8 & -0.51 & 0.204 \\
\hline Blue-gray Gnatcatcher & 0 & 0 & 0 & 0 & 0 & 0 & 0 & 0 & 0.8 & 0 & & \\
\hline Eastern Bluebird & 6.4 & 9.6 & 9.6 & 6.4 & 3.2 & $\mathbf{0}$ & $\mathbf{0}$ & $\mathbf{0}$ & $\mathbf{0}$ & $\mathbf{0}$ & -1.18 & 0.001 \\
\hline Gray Catbird & 0 & 1.6 & 0 & 9.6 & 12.8 & 9.6 & 8 & 4.8 & 0.8 & 0 & 0.06 & 0.915 \\
\hline Northern Mockingbird & 7.2 & 9.6 & 4.8 & 4.8 & 14.4 & $\mathbf{0}$ & $\mathbf{0}$ & $\mathbf{0}$ & $\mathbf{0}$ & $\mathbf{0}$ & -1.12 & 0.034 \\
\hline Brown Thrasher & 2.4 & 2.4 & 2.4 & 8 & 6.4 & 9.6 & 6.4 & 3.2 & 0 & 4.8 & 0.04 & 0.904 \\
\hline Pine Warbler & $\mathbf{0}$ & $\mathbf{0}$ & $\mathbf{0}$ & $\mathbf{0}$ & $\mathbf{0}$ & $\mathbf{0}$ & $\mathbf{0}$ & 1.6 & 3.2 & 4.8 & 0.45 & 0.007 \\
\hline Prairie Warbler & 23.2 & 14.4 & 12 & 19.2 & 30.4 & 32 & 17.6 & 12.8 & 12.8 & 3.2 & -1.15 & 0.257 \\
\hline Common Yellowthroat & 0.8 & 4 & 0 & 8 & 0 & 6.4 & 8 & 0 & 0 & 0 & -0.17 & 0.679 \\
\hline Yellow-breasted Chat & 10.4 & 14.4 & 20.8 & 12.8 & 14.4 & 12.8 & 20.8 & 16 & 9.6 & 6.4 & -0.43 & 0.425 \\
\hline Summer Tanager & 4 & 1.6 & 4 & 0 & 0 & 0 & 0 & 0 & 0.8 & 0 & & \\
\hline Eastern Towhee & 16 & 8.8 & 8.8 & 24 & 30.4 & 16 & 24 & 16 & 14.4 & 6.4 & -0.15 & 0.867 \\
\hline Bachman's Sparrow & 19.2 & 10.4 & 8 & 6.4 & 19.2 & 12.8 & $\mathbf{0}$ & $\mathbf{0}$ & $\mathbf{0}$ & $\mathbf{0}$ & -1.89 & 0.014 \\
\hline Chipping Sparrow & 3.2 & 0 & 0 & 0 & 0 & 0 & 0 & 0 & 0 & 0 & & \\
\hline Field Sparrow & 0 & 0 & 0 & 0 & 0 & 0 & 14.4 & 6.4 & 6.4 & 0 & 0.42 & 0.439 \\
\hline Northern Cardinal & 6.4 & 2.4 & 4.8 & 6.4 & 6.4 & 12.8 & 6.4 & 4.8 & 8 & 8 & 0.36 & 0.246 \\
\hline Blue Grosbeak & 16.8 & 4 & 11.2 & 8 & 16 & 6.4 & 12.8 & 6.4 & 6.4 & 6.4 & -0.58 & 0.26 \\
\hline Indigo Bunting & 24 & 14.4 & 13.6 & 9.6 & 54.4 & 38.4 & 16 & 6.4 & 6.4 & 12.8 & -1.15 & 0.532 \\
\hline Brown-headed Cowbird & 0 & 4.8 & 0 & 0 & 0 & 0 & 0 & 0 & 0 & 3.2 & & \\
\hline Orchard Oriole & 19.2 & 8 & 4.8 & 6.4 & 11.2 & $\mathbf{0}$ & $\mathbf{0}$ & $\mathbf{0}$ & $\mathbf{0}$ & $\mathbf{0}$ & -1.71 & 0.005 \\
\hline Total Species & 30 & 27 & 23 & 27 & 19 & 20 & 15 & 15 & 17 & 14 & -1.75 & 0.001 \\
\hline
\end{tabular}




\section{Conclusions}

Compared to the BBS and Clariant (Sandoz) data, there have been very few changes in the breeding bird populations on the forested BBC plots at SRS. Other than periodic burning on some of these plots, there has been very little change in the habitat characteristics of the plots over the years. Given this relative habitat stability, one might expect few changes in breeding bird populations. However, the limited number of census years and the use of different observers over the years probably limit the ability of the statistical tests to detect differences. Additional censuses will be required to improve sample sizes for future analyses.

The Clear-cut/Longleaf Pine Regeneration plot experienced significant habitat change over the years as the pines have matured. The population changes on the plot are consistent with the successional changes that have occurred. The data set for the plot consists of 10 years of continuous data, which probably also improves the ability of the statistical analyses to detect changes. Compared to the forested BBC plots, this larger data set produced a greater number of significant population changes than were observed on all of the forested plots combined. Additional censuses of this plot should be conducted to continue monitoring changes in breeding populations as the plot continues to mature.

\section{Acknowledgements}

The work at SRS would not have possible without the assistance of several field observers who conducted BBCs and point counts on FIA plots: Dale Droge , Michael Guzy, Chérie A. Irby, and Jonathan Plissner. Although William Jarvis was responsible for contracting much of the census work for the U. S. Forest Service, he often assisted our efforts by providing 
very valuable logistical support. In later years Laurel A Moore of the U. S. Forest Service at SRS assisted us greatly. We appreciate her patience while we prepared this final report.

We thank Dr. Hoke Hill of Clemson University who helped with the preliminary analyses of the Sandoz bird census data. Additional analyses of these data were completed by Carroll G. Belser of the Department of Biological Sciences at Clemson University, and we appreciate her efforts. 


\section{Literature Cited}

Brittingham, M. C. and S. A. Temple. 1983. Have cowbirds caused forest songbirds to decline? Bioscience 33: 21-25.

Faaborg, J. 2002. Saving migrant birds: Developing strategies for the future. University of Texas Press: Austin. 244 pp.

Faaborg, J. and W. J. Arendt. 1989. Long-term declines in winter resident warblers in a Puerto Rican dry forest. J. Wildl. Manage. 42: 1226-1229.

Finch, D. M. and P. W. Stangel (eds.). 1993. Status and management of Neotropical migratory birds. Proceedings of National Training Workshop, Estes Park, CO, September 21-25, 1992. General Technical Report RM-229, USDA Forest Service, Rocky Mountain Forest and Range Experiment Station, Fort Collins, CO. 422 pp.

Hagan, J. M. III and D. W. Johnson (eds.). 1992. Ecology and conservation of Neotropical migrant landbirds. Smithsonian Institution Press, Washington, D. C. 609 pp.

Hamel, P. B. 1992. Land manager's guide to the birds of the South. The Nature Conservancy, Southeastern Region, Chapel, NC. 437 pp. 
Hutto, R. L. 1988. Is tropical deforestation responsible for the reported decline in Neotropical migrant populations? American Birds 42(3): 375-379.

Sauer, J. R., J. E. Hines, and J. Fallon. 2003. The North American Breeding Bird Survey, Results and Analysis 1966 - 2002. Version 2003.1, USGS Patuxent Wildlife Research Center, Laurel, MD

Kilgo, J. C., K. E. Franzreb, S. A. Gauthreaux, Jr., K. V. Miller and B. R. Chapman. 2000. Effects of long-term forest management on a regional avifauna. Studies in Avian Biology 21: 8186.

McClure, J. P., N. D. Cost, and H. A. Knight. 1979. Multiresource inventories - A new concept for forest survey. USDA Forest Service Research Paper SE-191. 68 pp.

Morton, E. S. and R. Greenberg. 1989. The outlook for migratory songbirds: "future shock" for birders. American Birds 43: 1278-1283.

Peterjohn, B. G. and J. R. Sauer. 1994. Population trends of woodland birds from the North American breeding bird survey. Wildl. Soc. Bull. 22: 155-164.

Ralph, C. J., G. R. Geupel, P. Pyle, T. E. Martin, and D. F. DeSante. 1993. Handbook of field methods for monitoring landbirds. Gen. Tech. Rep. PSW-GTR-144. Albany, CA: Pacific Southwest Research Station, Forest Service, U. S. Dept. of Agriculture; 41 pp. 
Ralph, C. J., S. Droege, and J. R. Sauer. 1995. Managing and monitoring birds using point counts: standards and applications. In: Ralph, C. J., J. R. Sauer and S. Droege (eds.), Monitoring Bird Populations by Point Counts. Gen. Tech. Rep. PSW-GTR-149. Albany, CA: Pacific Southwest Research Station, Forest Service, U. S. Dept. of Agriculture; 187 pp.

Robbins, C. S., J. R. Sauer, R. S. Greenberg, and S. Droege. 1989. Population declines in North American birds that migrate to the neotropics. Proceedings of the National Academy of Sciences, USA 86: 7658-7662.

Sauer, J. R., B. G. Peterjohn, and W. A. Link. 1994. Observer differences in the North American Breeding Bird Survey. Auk 111:50-62.

Sheffield, R. M. 1981. Multiresource inventories: Techniques for evaluating nongame bird habitat. USDA Forest Service Research Paper SE-218, 28 pp.

Terborgh, J. 1989. Where have all the birds gone? Princeton University Press, 107 pp.

United States Department of Agriculture, Forest Service, Southern Region. 1988.

Silvicultural Examination and Prescription Field Book. 35 pp. and 8Stand Tally Sheets.

White, D. L. and K. F. Gaines. 2000. The Savannah River Site: site description, land use and management history. Studies in Avian Biology 21: 8-17. 
Wilcove, D. S. 1988. Changes in the avifauna of the Great Smoky Mountains: 1947-1983. Wilson Bulletin 100: 256-271. 


\section{Appendix I}

\section{Breeding Bird Census Plot Descriptions}

Bottomland Hardwood Forest - Established in1992. (J. Field Ornithol. Vol. 64, No.1 (Supplement): 55-56)

Description of Plot: A square plot (350 x 350 m) with a closed canopy dominated by Red Maple, Sweetgum, and Laurel Oak, estimated to be between 31 and 60 years of age. Mean canopy height, $20 \mathrm{~m}$ (range 15-20 m). The E three-quarters of the plot is dominated by a thick Cane understory. Other understory plants include Sweetgum and Red Maple. Three small $(<20 \mathrm{~m}$ diameter) openings in the canopy are scattered in the $\mathrm{N}$ half of the plot and are dominated by a thick ground cover of grape vines. Other ground cover plants include Cat Brier and Sphagnum. Approximately $50 \%$ of the plot was inundated during three visits, while $<2 \%$ was under water during the other six visits. The E 10-40 m of the plot is located above a $1.5 \mathrm{~m}$ embankment and contains vegetation more characteristic of drier hardwood forests. Edge: Up to 75\% of the plot's perimeter is bordered by the same habitat, and the plot lies within a tract of similar habitat 51100 ha in size. The narrow strip of drier hardwoods on the $\mathrm{E}$ edge is bordered by mature and regenerative pine plantations. Topography and Elevation: The plot is nearly level with a Wfacing slope of $<5 \%$. Minimum elevation $38 \mathrm{~m}$, maximum $43 \mathrm{~m}$. 
Clear-cut-Longleaf Pine Regeneration - Established in1992. (J. Field Ornithol. Vol. 64, No.1 (Supplement): 68-69)

Description of Plot: A rectangular plot (300 x 700 m). The plot was cleared in 1990 and Longleaf Pine seedlings were planted. There is no canopy except for a 0.5 ha area where a Hackberry and Southern Red Oak copse was left standing. A number of snags occur throughout the plot (2-3 per ha with several dense clumps). The limited understory is dominated by Black Cherry and Southern Red Oak. About 30\% of the plot consists of a dense growth of blackberry. Little Bluestem and Switchgrass make up much of the rest of the relatively sparse ground cover. Edge: Less than 25\% of the plot's perimeter is bordered by the same habitat, and more than 200 ha of similar habitat border on or are within a short distance of the plot. The NE and NW sections of the plot are bordered by Loblolly Pine forest. Isolated stands of mixed hardwoods border much of the $\mathrm{N}$ boundary. A Forest Service road runs along the $\mathrm{S}$ boundary and the roadside opposite the plot consists of a narrow band of dense young pines and cedars. A power line with steel towers runs along the W edge. Topography and Elevation: The plot is nearly level with a slope of $<5 \%$. Minimum elevation 85 m, maximum $92 \mathrm{~m}$.

1995 Census (J. Field Ornithol. Vol. 67, No. 4 (Supplement): 89-90)

Description of Plot: Because the site was initially clear-cut and replanted, the vegetation demonstrates growth and infilling each year. Planted longleaf pines are now approximately 1.2$1.5 \mathrm{~m}$ tall. Clumps of naturally-occurring trees, such as sweetgum, and various shrubs have become dense in some areas. Vertical structure of the vegetation has significantly increased since plot organization. Because vegetation presently inhibits identification of bird species to $100 \mathrm{~m}$, 
plot size was reduced from 25 to 12.5 ha. Grid intersections were likewise reduced from $100 \mathrm{~m}$ to $50 \mathrm{~m}$.

\section{Census}

Description of Plot: Because the site was initially clearcut and replanted, the vegetation demonstrates growth and infilling each year. Planted longleaf pines are now approximately 4-10 $\mathrm{m}$ tall. Over most of the plot the ground cover is sparse. Blackberry (Rubus sp.) is common throughout the plot. There are also isolated clumps of dense vegetation (sweetgum, privet, and other shrubs). The largest of these is approximately 0.25 ha. The plot was burned since the 2000 census, reducing the amount of shrub and ground cover compared to 1999 and 2000. 
Loblolly Pine Forest (Loblolly II) - Established in1993. (J. Field Ornithol. Vol. 65, No.2 (Supplement): 75)

Description of Plot: A rectangular plot (350 x 400 m) in a 34-yr-old stand located in the SE section of the Savannah River Site. The closed canopy is dominated by loblolly pine. There are also 2-3 clumps of water oaks with maximum heights below the canopy of loblolly pine, and some sweetgum. Mean canopy height, $18 \mathrm{~m}$ (range 6-20 m). The minimal understory is dominated by sweetgum, wax myrtle, and red maple. The sparse ground cover is dominated by muscadine, yellow jessamine, and gooseberry. Edge: At least $60 \%$ of the plot's perimeter is bordered by the same habitat, and the plot is part of a stand 48.2 ha in size. The SE edge is bordered by a Forest Service road on an old railroad bed with similar habitat across the road. The SW corner is bordered by a road and hardwoods, with a small stream located across the road. Topography and Elevation: The plot is nearly level with a slope of $<5 \%$. Minimum elevation 73 m, maximum 82m. 
Loblolly Pine Plantation (cut in 1981, Loblolly I) -- Established in 1995. (J. Field Ornithol. Vol. 67 (Supplement): 58)

Description of Plot: A square plot (350 X 350 m) located in the SW section of the Savannah River Site. A seed-tree cut was performed in 1981. Scattered remnant loblolly pine trees were left standing for reseeding the site. The forest has regenerated with loblolly trees of approximately uniform size (7.6-9.1 $\mathrm{m}$ tall with the exception of the larger seed trees which are up to $17 \mathrm{~m}$ tall). The young trees currently form a low, thick canopy. A hardwood inclusion, dominated by understory-sized water oaks, is located in the $\mathrm{N}$ section of the plot. Clumps of water oak saplings are scattered throughout the $\mathrm{N}$ section. The ground cover is sparse and dominated by poison oak, wax myrtle, and yellow jessamine. Edge: More than $75 \%$ of the plot's perimeter is bordered by the same habitat, and the plot lies within a tract of similar habitat $<25$ ha in size. The $\mathrm{N}$ border of the plot parallels a train track and a dirt access road. The plot boundary is set back approximately $10 \mathrm{~m}$ from the dirt road to avoid inclusion of edge habitat. North of the train track is a clear-cut. The young loblolly plantation extends to the $\mathrm{S}$ and $\mathrm{W}$ of the plot. However, the stand is not much larger than the plot itself. A small, depressional, mature hardwood forest borders part of the $\mathrm{E}$ edge of the plot. Main roads border the mature hardwoods to the E and the loblolly pine plantation to the S. Topography and Elevation: The plot is nearly level with a SE-facing slope of $<5 \%$ grade. Minimum elevation $62 \mathrm{~m}$, maximum $71 \mathrm{~m}$. 
Longleaf Pine Forest - Established in1992. (J. Field Ornithol. Vol. 64, No.1 (Supplement): 6869)

Description of Plot: A square plot (350 x 350 m). A canopy dominated by mature Longleaf Pine, estimated to be between 31 and 60 years of age, covers $80 \%$ of the plot. Mean canopy height, $22 \mathrm{~m}$ (range 15-25 m). Where there is no canopy the understory is dominated by Sassafras, Laurel Oak, and Common Persimmon, and the ground cover is dominated by Poison Oak, Deer Berry, Camphor Weed, Little Bluestem, and Aristida grasses. The U. S. Forest Service uses a fire rotation schedule to manage the habitat. Edge: Up to 75\% of the plot's perimeter is bordered by the same habitat, and the plot lies within a tract of similar habitat 51100 ha in size. A lightly traveled Forest Service road runs along the N boundary. Topography and Elevation: The plot is nearly level with a slope of $<5 \%$. Minimum elevation $79 \mathrm{~m}$, maximum $82 \mathrm{~m}$. 
Scrub Oak Forest with Scattered Longleaf Pine - Established in1993. (J. Field Ornithol. Vol. 65, No.2 (Supplement): 94)

Description of Plot: A square plot (350 x 350 m) located in the NE corner of the Savannah River Site and maintained by the U. S. Forest Service. The open canopy is dominated by turkey oak, longleaf pine, and bluejack oak, estimated to be between 31 and 60 years of age. The mature longleaf pines are scattered throughout, rising well above the oak canopy. Mean canopy height, 7 $\mathrm{m}$ (range 5-15 m). The uniformly dense understory is dominated by turkey oak, sparkleberry, and dwarf post oak. The variable ground cover is dominated by gooseberry and dwarf huckleberry in the $\mathrm{S}$ and $\mathrm{W}$ portions of the plot, and by patches of bracken fern in the $\mathrm{E}$ half. Ground cover is sparsely distributed in much of the $\mathrm{N}$ section. The substrate is xeric and sandy, with no streams or standing water present. Edge: The plot is bordered on all sides by similar habitat, although a clear-cut area is present across a woods road running tangential to the SW corner. An 18-ha Department of Energy set aside area borders the W edge. The plot lies within a tract of similar habitat 26-50 ha in size. Topography and Elevation: The plot is nearly level with a S-facing slope of $<5 \%$. Minimum elevation $93 \mathrm{~m}$, maximum $108 \mathrm{~m}$. 
Tupelo-Cypress Swamp - Established in1992. (J. Field Ornithol. Vol. 64, No.1 (Supplement): 80-81)

Description of Plot: A rectangular plot (200 x 550 m) with a closed canopy dominated by Water Tupelo and Bald Cypress, estimated to be between 61 and 100 years of age. Mean canopy height, $25 \mathrm{~m}$ (range 18-35 m). The limited understory is also dominated by Water Tupelo and Bald Cypress. Most of the patchily distributed ground cover consists of emergent vegetation such as Slender Arrowhead and beak rush. Other plants, such as Virginia-willow, Poison Ivy, and various briers, sprout along fallen logs. The plot is completely inundated throughout the year and is homogeneous in both vegetative composition and structure. No channels exist, but there is a constant ESE flow of water. Three small $(<25 \mathrm{~m})$ "ponds" are present where the canopy is open, one of which contains vegetation (water lilies) not located elsewhere on the plot. Remnant cypress stumps measuring $>2 \mathrm{~m}$ in diameter are scattered throughout, but no living trees exceed $1.2 \mathrm{~m}$ dbh. Edge: Up to $75 \%$ of the plot's perimeter is bordered by the same habitat, and the plot lies within a tract of similar habitat $>500$ ha in size. The NNE border of the plot is located along the edge of the swamp and consists of a strip of upland hardwood forest 15-200 m wide. Managed pine woodlands and clear-cut areas are located beyond the hardwoods. The swamp extends 1-1.5 km beyond the SSW border of the plot to the Savannah River and continues both upstream (WNW) and downstream (ESE) for several km. Topography and Elevation: The plot is level. Minimum elevation $26 \mathrm{~m}$, maximum $26 \mathrm{~m}$. 
Upland Hardwood Forest - Established in1992. (J. Field Ornithol. Vol. 64, No.1 (Supplement): 57)

Description of Plot: A square plot (350 x 350 m). A closed canopy dominated by Southern Red Oak, Mockernut Hickory, and White Oak, estimated to be between 61 and 100 years of age, covers $80 \%$ of the plot. Mean canopy height, $25 \mathrm{~m}$ (range 18-30 m). The understory is dominated by Flowering Dogwood, American Holly, and Sweetgum. The ground cover is dominated by grape, Cane, and Butterfly-Pea. A small spring runs the length of the plot, $100 \mathrm{~m}$ from the south edge. The more hydric soils along the streams support vegetation such as Cane, which is not found in other areas of the plot. Edge: Less than $25 \%$ of the plot's border perimeter is bordered by the same habitat, and the plot lies within a tract of similar habitat 26-50 ha in size. A large stream (4-8 $\mathrm{m}$ wide) borders the $\mathrm{W}$ and half of the $\mathrm{N}$ edges. The $\mathrm{S}$ border is $3-15 \mathrm{~m}$ from the edge of a grass-forb-dominated power line corridor. Topography and Elevation: Most of the land slopes $20-40^{\circ}$ toward the spring that runs through the plot. A similar slope is located along the W $50 \mathrm{~m}$ of the plot, facing the stream. Within $50 \mathrm{~m}$ of two-thirds of the $\mathrm{N}$ edge, the land drops 18-20 m along a wooded 45-60 slope. Minimum elevation 49 m, maximum 76 m. 
Appendix II

Breeding Bird Census Plot Census Data 
Bottomland Hardwood Plot Census Data. Numbers are territories/40 ha.

\begin{tabular}{|c|c|c|c|c|c|c|c|c|}
\hline Year & 1992 & 1993 & 1994 & 1995 & 1996 & 1997 & 1998 & 1999 \\
\hline Observer & $\mathrm{JP}$ & & $\mathrm{CI}$ & & MG & & & SW \\
\hline Plot area (ha) & 12.2 & & 12.2 & & 12.2 & & & 12.2 \\
\hline Census Trips & 9 & & 10 & & 10 & & & 10 \\
\hline \multicolumn{9}{|l|}{ Cooper's Hawk } \\
\hline Red-shouldered Hawk & 1.6 & & $\mathrm{v}$ & & 0.0 & & & 0.0 \\
\hline Red-tailed Hawk & 0.0 & & 0.0 & & $\mathrm{v}$ & & & 0.0 \\
\hline Wild Turkey & 0.0 & & 0.0 & & $\mathrm{v}$ & & & 0.0 \\
\hline \multicolumn{9}{|l|}{ Northern Bobwhite } \\
\hline Mourning Dove & 0.0 & & 4.9 & & 0.0 & & & 0.0 \\
\hline \multicolumn{9}{|l|}{ Common Ground-dove } \\
\hline Yellow-billed Cuckoo & 3.3 & & 6.6 & & 8.2 & & & 9.8 \\
\hline Eastern Screech-owl & 0.0 & & 0.0 & & $\mathrm{v}$ & & & 0.0 \\
\hline Barred Owl & 0.0 & & 0.0 & & $\mathrm{v}$ & & & $\mathrm{v}$ \\
\hline Barn Owl & 0.0 & & $\mathrm{v}$ & & 0.0 & & & 0.0 \\
\hline
\end{tabular}

Common Nighthawk

Chuck-will's-widow

Whip-poor-will

Chimney Swift

Ruby-throated

Hummingbird

$\begin{array}{llll}0.0 & 0.0 & 3.3 & 3.3\end{array}$

Red-headed Woodpecker

Red-bellied Woodpecker

Downy Woodpecker

$6.6 \quad 6.6$

Northern Flicker

$6.6 \quad 4.9$

$0.0 \quad 0.0$

6.2
4.95

8.2 *

Pileated Woodpecker $\quad 3.3$

Eastern Wood-Pewee $\quad 0.0$

Acadian Flycatcher $\quad 18.0$

0.0

6.6

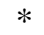

1.6

$\mathrm{v} \quad 0.0$

Great Crested Flycatcher $\quad 4.9$

Eastern Kingbird $\quad 0.0$

White-eyed Vireo $\quad 13.1$

Yellow-throated Vireo $\quad 3.3$

Red-eyed Vireo

Blue Jay

American Crow

Fish Crow

Purple Martin

Barn Swallow

Carolina Chickadee

Tufted Titmouse

White-breasted Nuthatch

29.5

$\mathrm{V}$

0.0

0.0

0

$\mathrm{V}$

3.3

0.0

23.0

23.0

0.0

0.0

6.6

27.9

27.9

$\begin{array}{lll}6.6 & 6.6 & 0.0\end{array}$

0.0

0.0

0.0

$\begin{array}{lll}0.0 & 16.4 & \mathrm{~V}\end{array}$

$\mathrm{v}$

0.0

$\mathrm{v}$

$37.7-23.0$

37.7

37.7

23.0

$\mathrm{v}$

0.0

$\mathrm{v}$

$\mathrm{V}$

0.0

0.0

$0.0 \quad \mathrm{v}$

9.8

0.0

6.6

3.3

9.8

6.6

11.5

8.2

$\mathrm{v}$

0.0

$\mathrm{v}$

Brown-headed Nuthatch

Carolina Wren

19.7

13.1

18.0

14.8

Blue-gray Gnatcatcher

9.8

11.5

21.3

21.3 


\section{Bottomland Hardwood Plot Census Data (cont.)}

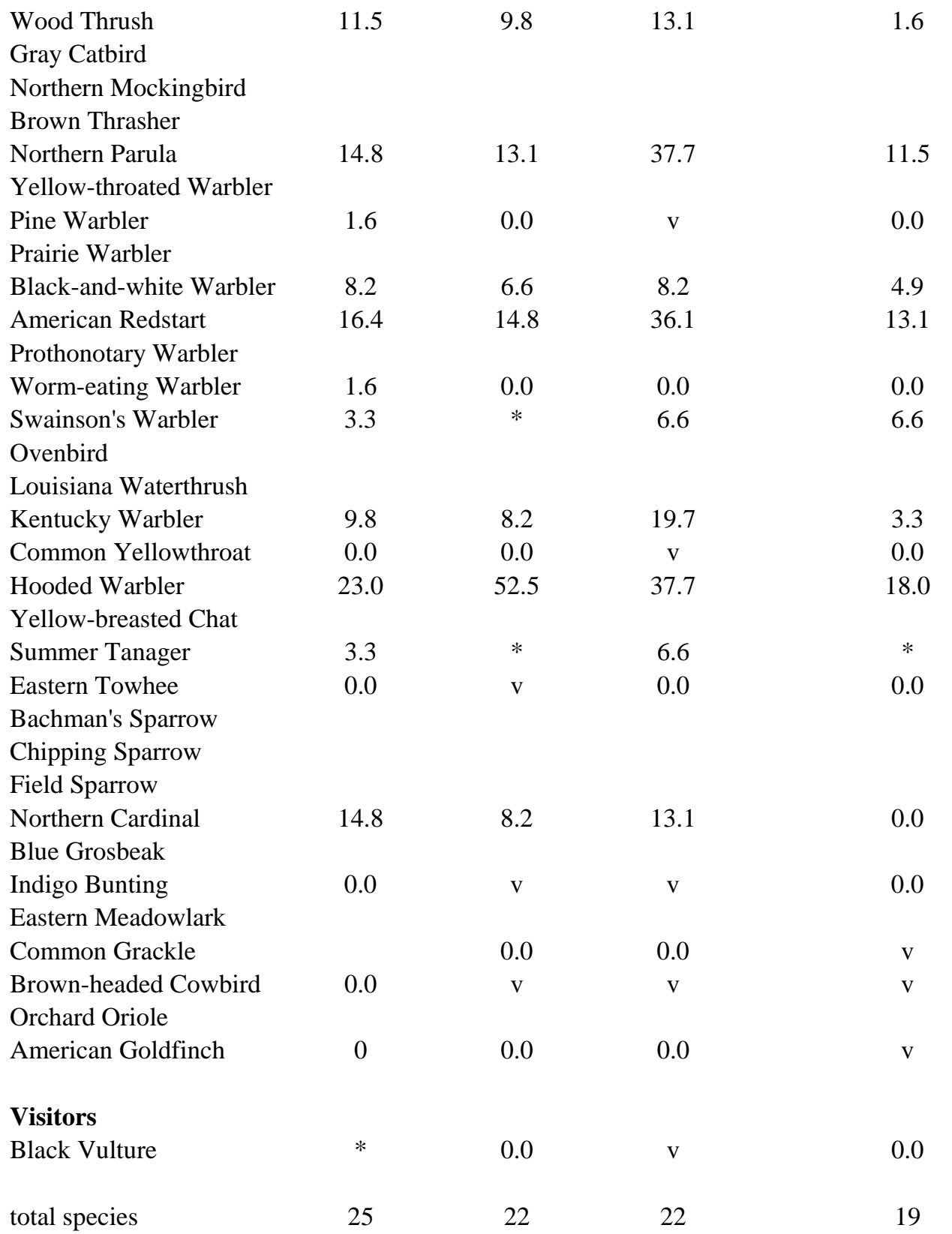


Clear-cut-Longleaf Pine Regeneration Plot Census Data. Numbers are territories/40 ha.

$*=<0.5$ territory on plot, $\mathrm{v}=$ visitor

\begin{tabular}{|c|c|c|c|c|c|c|c|c|c|c|}
\hline Year & 1992 & 1993 & 1994 & 1995 & 1996 & 1997 & 1998 & 1999 & 2000 & 2001 \\
\hline Observer & DD & SW & CI & CI & MG & MG & SW & SW & SW & SW \\
\hline Plot area (ha) & 25 & 25 & 25 & 12.5 & 12.5 & 12.5 & 12.5 & 12.5 & 12.5 & 12.5 \\
\hline Census Trips & 8 & 8 & 10 & 10 & 10 & 10 & 10 & 10 & 10 & 10 \\
\hline Stand Age (years) & 2 & 3 & 4 & 5 & 6 & 7 & 8 & 9 & 10 & 11 \\
\hline Cooper's Hawk & 0 & 0 & 0 & 0 & $\mathrm{v}$ & 0 & 0 & 0 & 0 & 0 \\
\hline Red-shouldered Hawk & 0 & & & & & & & & & \\
\hline Red-tailed Hawk & 0 & 0 & 0 & 0 & $\mathrm{v}$ & 0 & 0 & 0 & 0 & 0 \\
\hline \multicolumn{11}{|l|}{ Wild Turkey } \\
\hline Northern Bobwhite & 20 & 4.8 & 9.6 & 9.6 & 0 & $\mathrm{v}$ & 8 & 0 & 0 & 0 \\
\hline Rock Dove & 0 & 0 & 0 & 0 & $\mathrm{v}$ & 0 & 0 & 0 & 0 & 0 \\
\hline Mourning Dove & 12.8 & 0 & 11.2 & 12.8 & $\mathrm{~V}$ & $\mathrm{~V}$ & 0 & 3.2 & 3.2 & 3.2 \\
\hline Common Ground-dove & 3.2 & 4 & 4.8 & 3.2 & 6.4 & 3.2 & 4.8 & 0 & 0 & 0 \\
\hline Yellow-billed Cuckoo & 0.8 & 0 & 0 & 0 & 0 & 0 & 0 & 0 & 0 & 0 \\
\hline \multicolumn{11}{|l|}{ Eastern Screech-Owl } \\
\hline & \multicolumn{7}{|c|}{$\begin{array}{l}\text { Chuck-will's-widow } \\
\text { Whip-poor-will }\end{array}$} & & 0 & 0 \\
\hline \multicolumn{11}{|l|}{$\begin{array}{l}\text { Ruby-throated } \\
\text { Hummingbird }\end{array}$} \\
\hline Red-headed Woodpecker & 3.2 & 4 & 4.8 & 4.8 & $\mathrm{v}$ & 0 & 0 & 0 & 0 & 0 \\
\hline Red-bellied Woodpecker & 2.4 & 2.4 & 4.8 & 3.2 & 3.2 & $*$ & 0 & 0 & 0 & 0 \\
\hline Downy Woodpecker & $*$ & 0 & $\mathrm{v}$ & 1.6 & $\mathrm{v}$ & 0 & 0 & 0 & 0 & 0 \\
\hline Northern Flicker & 3.2 & 1.6 & 2.4 & 3.2 & $\mathrm{v}$ & $\mathrm{v}$ & 0 & 0 & 0 & 0 \\
\hline Pileated Woodpecker & 0 & 0 & $\mathrm{~V}$ & 0 & 0 & $\mathrm{v}$ & 0 & 0 & 0 & 0 \\
\hline Eastern Wood-Pewee & 0 & 0 & $\mathrm{v}$ & 0 & 0 & 0 & 0 & 0 & 0 & 0 \\
\hline \multicolumn{11}{|l|}{ Acadian Flycatcher } \\
\hline Eatern Phoebe & 0 & 0 & $\mathrm{v}$ & 0 & 0 & 0 & 0 & 0 & 0 & 0 \\
\hline Great Crested Flycatcher & 3.2 & 2.4 & 4 & 3.2 & 6.4 & 3.2 & 0 & 0 & 3.2 & 0 \\
\hline Eastern Kingbird & 4.8 & 2.4 & 4 & 6.4 & 4.8 & $*$ & 0 & 0 & 0 & 0 \\
\hline White-eyed Vireo & 4.8 & 0.8 & 3.2 & 3.2 & 0 & 4.8 & 11.2 & 11.2 & 9.6 & 6.4 \\
\hline \multicolumn{11}{|l|}{ Yellow-throated Vireo } \\
\hline \multicolumn{11}{|l|}{ Red-eyed Vireo } \\
\hline Blue Jay & 1.6 & 0 & 0 & 1.6 & $\mathrm{v}$ & $*$ & 0 & 0.8 & 0 & 1.6 \\
\hline American Crow & 0 & 0 & 0 & $\mathrm{v}$ & $\mathrm{v}$ & $\mathrm{v}$ & 0 & 0 & 0 & 0 \\
\hline Fish Crow & 0 & 0 & 0 & $\mathrm{v}$ & $\mathrm{v}$ & $\mathrm{v}$ & 0 & 0 & 0 & 0 \\
\hline Purple Martin & 0 & 0 & 0 & $\mathrm{v}$ & 0 & 0 & 0 & 0 & 0 & 0 \\
\hline Barn Swallow & 0 & 0 & 0 & 0 & 0 & $\mathrm{v}$ & 0 & 0 & 0 & 0 \\
\hline Carolina Chickadee & 0 & 0.8 & $\mathrm{v}$ & 0 & 9.6 & 6.4 & 3.2 & 1.6 & 3.2 & 3.2 \\
\hline Tufted Titmouse & 0.8 & 0 & $\mathrm{v}$ & 0 & $\mathrm{v}$ & 0 & 0 & 0 & 3.2 & 0 \\
\hline White-breasted Nuthatch & 0 & 0 & 0 & $\mathrm{v}$ & 0 & 0 & 0 & 0 & 0 & 0 \\
\hline Brown-headed Nuthatch & 0.8 & 0 & $\mathrm{v}$ & 3.2 & $\mathrm{v}$ & 0 & 0 & 0 & 0 & 0 \\
\hline Carolina Wren & 9.6 & 4.8 & 6.4 & 3.2 & 12.8 & 8 & 6.4 & 1.6 & 1.6 & 4.8 \\
\hline
\end{tabular}




\section{Clear-cut-Longleaf Pine Regeneration Plot Census Data (cont.)}

$\begin{array}{lcccccccccc}\text { Blue-gray Gnatcatcher } & 0 & 0 & \mathrm{v} & \mathrm{v} & \mathrm{v} & 0 & 0 & 0 & 0.8 & 0 \\ \text { Eastern Bluebird } & 6.4 & 9.6 & 9.6 & 6.4 & 3.2 & * & 0 & 0 & 0 & 0 \\ \text { Wood Thrush } & & & & & & & & & & \\ \text { Gray Catbird } & 0 & 1.6 & \mathrm{v} & 9.6 & 12.8 & 9.6 & 8 & 4.8 & 0.8 & 0 \\ \text { Northern Mockingbird } & 7.2 & 9.6 & 4.8 & 4.8 & 14.4 & \mathrm{v} & 0 & 0 & 0 & 0 \\ \text { Brown Thrasher } & 2.4 & 2.4 & 2.4 & 8 & 6.4 & 9.6 & 6.4 & 3.2 & 0 & 4.8 \\ \text { Northern Parula } & & & & & & & & & & \\ \text { Yellow-throated Warbler } & & & & & & & & & & \\ \text { Pine Warbler } & 0 & 0 & 0 & 0 & 0 & 0 & 0 & 1.6 & 3.2 & 4.8 \\ \text { Prairie Warbler } & 23.2 & 14.4 & 12 & 19.2 & 30.4 & 32 & 17.6 & 12.8 & 12.8 & 3.2 \\ \text { Black-and-white Warbler } & & & & & & & & & & \\ \text { American Redstart } & & & & & & & & & & \\ \text { Prothonotary Warbler } & & & & & & & & & & \\ \text { Worm-eating Warbler } & & & & & & & & & & \\ \text { Swainson's Warbler } & & & & & & & & & & \\ \text { Ovenbird } & & & & & & & & & & \\ \text { Louisiana Waterthrush } & & & & & & & & & & \\ \text { Kentucky Warbler } & & & & & & & & & \\ \text { Common Yellowthroat } & 0.8 & 4 & \mathrm{v} & 8 & \mathrm{v} & 6.4 & 8 & 0 & 0 & 0 \\ \text { Hooded Warbler } & & & & & & & & & & \\ \text { Yellow-breasted Chat } & 10.4 & 14.4 & 20.8 & 12.8 & 14.4 & 12.8 & 20.8 & 16 & 9.6 & 6.4 \\ \text { Summer Tanager } & 4 & 1.6 & 4 & \mathrm{v} & \mathrm{v} & \mathrm{v} & 0 & 0 & 0.8 & 0 \\ \text { Eastern Towhee } & 16 & 8.8 & 8.8 & 24 & 30.4 & 16 & 24 & 16 & 14.4 & 6.4 \\ \text { Bachman's Sparrow } & 19.2 & 10.4 & 8 & 6.4 & 19.2 & 12.8 & 0 & 0 & 0 & 0 \\ \text { Chipping Sparrow } & 3.2 & 0 & 0 & 0 & 0 & \mathrm{v} & 0 & 0 & 0 & 0 \\ \text { Field Sparrow } & 0 & 1.6 & 4 & 6.4 & 9.6 & 9.6 & 14.4 & 6.4 & 6.4 & 0 \\ \text { Northern Cardinal } & 6.4 & 2.4 & 4.8 & 6.4 & 6.4 & 12.8 & 6.4 & 4.8 & 8 & 8 \\ \text { Blue Grosbeak } & 16.8 & 4 & 11.2 & 8 & 16 & 6.4 & 12.8 & 6.4 & 6.4 & 6.4 \\ \text { Indigo Bunting } & 24 & 14.4 & 13.6 & 9.6 & 54.4 & 38.4 & 16 & 6.4 & 6.4 & 12.8 \\ \text { Eastern Meadowlark } & 4.8 & 1.6 & \mathrm{v} & 0 & 0 & 0 & 0 & 0 & 0 & 0 \\ \text { Common Grackle } & 0 & 0 & 0 & 0 & \mathrm{v} & 0 & 0 & 0 & 0 & 0 \\ \text { Brown-headed Cowbird } & 0 & 4.8 & \mathrm{v} & \mathrm{v} & \mathrm{v} & \mathrm{v} & 0 & 0 & 0 & 3.2 \\ \text { Orchard Oriole } & 19.2 & 8 & 4.8 & 6.4 & 11.2 & 0 & 0 & 0 & 0 & 0 \\ \text { American Goldfinch } & & & & & & & & & & \end{array}$

\section{Visitors}

Double-crested Cormorant

Great Blue Heron

Great Egret

Cattle Egret

Green Heron

White Ibis

Black Vulture

Turkey Vulture

total species

$\begin{array}{llllllllll}0 & 0 & 0 & 0 & \mathrm{v} & 0 & 0 & 0 & 0 & 0 \\ 0 & 0 & 0 & 0 & \mathrm{v} & 0 & 0 & 0 & 0 & 0 \\ 0 & 0 & 0 & 0 & \mathrm{v} & \mathrm{v} & 0 & 0 & 0 & 0 \\ 0 & 0 & 0 & 0 & \mathrm{v} & 0 & 0 & 0 & 0 & 0 \\ 0 & 0 & 0 & 0 & \mathrm{v} & 0 & 0 & 0 & 0 & 0 \\ 0 & 0 & 0 & \mathrm{v} & \mathrm{v} & \mathrm{v} & 0 & 0 & 0 & 0 \\ 0 & 0 & 0 & \mathrm{v} & \mathrm{v} & 0 & 0 & 0 & 0 & 0 \\ 30 & 27 & 23 & 27 & 19 & 20 & 15 & 15 & 17 & 14\end{array}$


Loblolly II Plot Census Data. Numbers are territories/40 ha.

$*=<0.5$ territory on plot, $\mathrm{v}=$ visitor

$\begin{array}{lcccccccc}\text { Year } & 1992 & 1993 & 1994 & 1995 & 1996 & 1997 & 1998 & 1999 \\ \text { Observer } & & \text { SW } & \text { CI } & & & \text { MG } & & \\ \text { Plot area (ha) } & & 12.2 & 12.2 & & & 12.2 & & \\ \text { Census Trips } & & 10 & 10 & & & 10 & & \end{array}$

Cooper's Hawk

Red-shouldered Hawk

Red-tailed Hawk

Wild Turkey

Northern Bobwhite

Mourning Dove

$\begin{array}{lll}\mathrm{v} & 3.3 & 0.0\end{array}$

Common Ground-dove

$\begin{array}{lll}0.0 & 1.6 & 0.0\end{array}$

Eastern Screech-Owl

Barred Owl

Common Nighthawk

Chuck-will's-widow

Whip-poor-will

Chimney Swift

Ruby-throated

Hummingbird

Red-headed Woodpecker

$3.3 \quad 6.6$

Red-bellied Woodpecker

$3.3 \quad 4.9$

3.3

Downy Woodpecker

Northern Flicker

Pileated Woodpecker

Eastern Wood-Pewee

Acadian Flycatcher

Great Crested Flycatcher

$\mathrm{V} \quad 4.9$

* $\quad 3.3$

$0.0 \quad 1.6$

$6.6 \quad 8.2$

3.3

$\begin{array}{lll}\mathrm{V} & \mathrm{V} & \mathrm{V}\end{array}$

Eastern Kingbird

White-eyed Vireo

$\begin{array}{lll}9.8 & 11.5 & 6.6\end{array}$

Yellow-throated Vireo

Red-eyed Vireo

Blue Jay

American Crow

Fish Crow

Purple Martin

Barn Swallow

Carolina Chickadee

Tufted Titmouse

White-breasted Nuthatch

Brown-headed Nuthatch

Carolina Wren

$\begin{array}{lll}0.0 & 1.6\end{array}$

$\begin{array}{lll}0.0 & 1.6 & 0.0\end{array}$

$\begin{array}{lll}\mathrm{v} & \mathrm{V} & 0.0\end{array}$

$\begin{array}{llll}\mathrm{v} & \mathrm{V} & \mathrm{v}\end{array}$

$\begin{array}{lll}0.0 & 0.0 & \mathrm{~V}\end{array}$

Blue-gray Gnatcatcher

Eastern Bluebird

$\begin{array}{lll}\mathrm{v} & 0.0 & 0.0\end{array}$

$\begin{array}{lll}\mathrm{v} & 1.6 & 3.3\end{array}$

$\begin{array}{lll}3.3 & 4.9 & 0.0\end{array}$

3.311 .5 *

$3.311 .5 \quad 6.6$

$\begin{array}{lll}\mathrm{V} & \mathrm{V} & 3.3\end{array}$ 


\section{Loblolly II Plot Census Data (cont.)}

Wood Thrush

Gray Catbird

Northern Mockingbird

Brown Thrasher

Northern Parula

Yellow-throated Warbler

Pine Warbler

$0.0 \quad \mathrm{~V}$

$*$

Prairie Warbler

$23.0 \quad 18.0$

23.0

Black-and-white Warbler

$0.0 \quad 0.0$

3.3

American Redstart

Prothonotary Warbler

Worm-eating Warbler

Swainson's Warbler

Ovenbird

$0.0 \quad \mathrm{~V}$

0.0

Louisiana Waterthrush

Kentucky Warbler

Common Yellowthroat

Hooded Warbler

Yellow-breasted Chat

Summer Tanager

Eastern Towhee

$8.2 \quad 6.6$

$3.3 \quad 8.2$

6.6

Bachman's Sparrow

Chipping Sparrow

$0.0 \quad 0.0$

$\mathrm{V}$

Field Sparrow

Northern Cardinal

$1.6 \quad 14.8$

6.6

Blue Grosbeak

Indigo Bunting

* $\quad 3.3$

8.2

Eastern Meadowlark

Common Grackle

Brown-headed Cowbird

Orchard Oriole

American Goldfinch

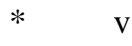

V

V $\quad 0.0$

V

Visitors

Wood Duck

V V

0.0

total species

$14 \quad 20$

18 
Loblolly I Plot Census Data. Numbers are territories/40 ha.

$*=<0.5$ territory on plot, $\mathrm{v}=$ visitor

$\begin{array}{lcccccccc}\text { Year } & 1992 & 1993 & 1994 & 1995 & 1996 & 1997 & 1998 & 1999 \\ \text { Observer } & & & & \text { CI } & & \text { MG } & & \text { SW } \\ \text { Plot area (ha) } & & & & 12.2 & 12.2 & & 12.2 \\ \text { Census Trips } & & & & 10 & & 10 & & 10\end{array}$

Cooper's Hawk

Red-shouldered Hawk

Red-tailed Hawk

Wild Turkey

Northern Bobwhite

$\begin{array}{lll}\mathrm{V} & 0.0 & 0.0\end{array}$

Mourning Dove

$\begin{array}{lll}\mathrm{v} & 3.3 & 0.0\end{array}$

Common Ground-dove

$\begin{array}{lll}\mathrm{V} & 0.0 & 3.3\end{array}$

Eastern Screech-Owl

Barred Owl

Common Nighthawk

0.0

$0.0 \quad \mathrm{v}$

Chuck-will's-widow

Whip-poor-will

Chimney Swift

Ruby-throated

Hummingbird

Red-headed Woodpecker

$\begin{array}{lll}0.0 & \mathrm{v} & 0.0\end{array}$

Red-bellied Woodpecker

$\mathrm{V}$

Downy Woodpecker

Northern Flicker

Pileated Woodpecker

Eastern Wood-Pewee

Acadian Flycatcher

Great Crested Flycatcher

Eastern Kingbird

White-eyed Vireo

Yellow-throated Vireo

Red-eyed Vireo

Blue Jay

American Crow

Fish Crow

3.28

$\mathrm{V}$

0.0

$*$

$\begin{array}{lll}6.56 & 3.3 & 3.3\end{array}$

$\begin{array}{lll}1.64 & 0.0 & 0.0\end{array}$

$\begin{array}{lll}1.64 & 0.0 & 0.0\end{array}$

$\begin{array}{lll}0.0 & \mathrm{v} & \mathrm{v}\end{array}$

$\begin{array}{lll}\mathrm{v} & 3.3 & \mathrm{v}\end{array}$

$\begin{array}{lll}\mathrm{V} & \mathrm{V} & 0.0\end{array}$

$\begin{array}{lll}0.0 & 0.0 & \mathrm{v}\end{array}$

Purple Martin

Barn Swallow

Carolina Chickadee

$\begin{array}{lll}3.28 & \mathrm{v} & 3.3\end{array}$

Tufted Titmouse

8.2

4.9

White-breasted Nuthatch

Brown-headed Nuthatch

Carolina Wren

Blue-gray Gnatcatcher

4.92

0.0

$\begin{array}{cc}\mathrm{V} & \mathrm{V} \\ \mathrm{V} & \mathrm{V} \\ \mathrm{V} & 0.0\end{array}$


Loblolly I Plot Census Data (cont.)

Wood Thrush

4.92

0.0

0.0

Gray Catbird

Northern Mockingbird

Brown Thrasher

V

1.64

V

0.0

Northern Parula

Yellow-throated Warbler

Pine Warbler

4.92

3.3

3.3

Prairie Warbler

Black-and-white Warbler

American Redstart

0.0

V

0.0

Prothonotary Warbler

Worm-eating Warbler

Swainson's Warbler

Ovenbird

Louisiana Waterthrush

Kentucky Warbler

Common Yellowthroat

Hooded Warbler

Yellow-breasted Chat

Summer Tanager

Eastern Towhee

0.0

0.0

$\mathrm{V}$

$\mathrm{V}$

Bachman's Sparrow

Chipping Sparrow

Field Sparrow

Northern Cardinal

9.84

9.8

6.6

Blue Grosbeak

0.0

0.0

$\mathrm{v}$

Indigo Bunting

Eastern Meadowlark

Common Grackle

Brown-headed Cowbird

V

V

Orchard Oriole

American Goldfinch

Visitors

Green Heron

0.0

$*$

V

total species

13

8

6 
Longleaf Plot Census Data. Numbers are territories/40 ha.

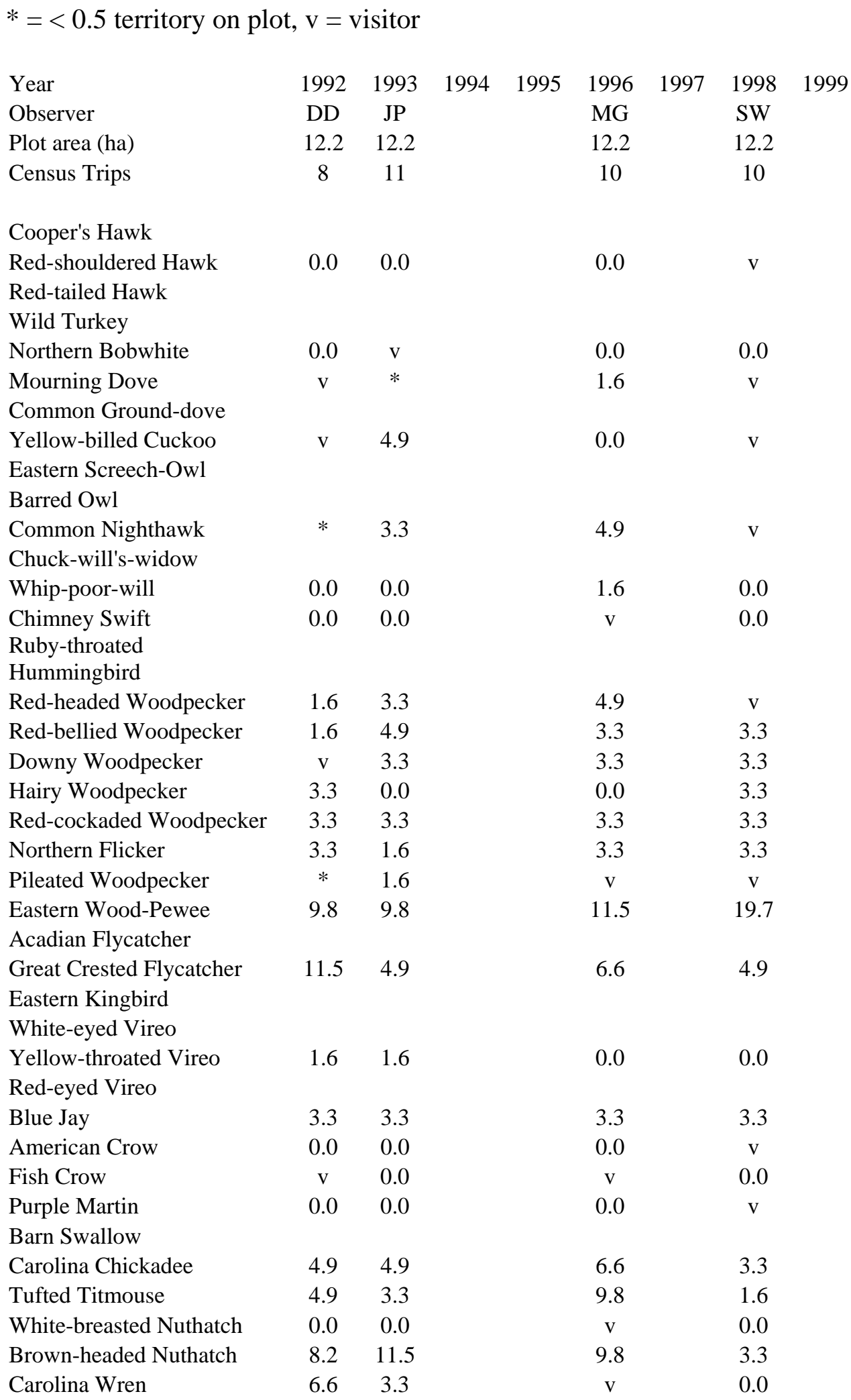




\section{Longleaf Plot Census Data (cont.)}

\begin{tabular}{|c|c|c|c|c|}
\hline Blue-gray Gnatcatcher & $*$ & 3.3 & $\mathrm{v}$ & 0.0 \\
\hline Eastern Bluebird & 6.6 & 8.2 & 3.3 & 4.9 \\
\hline Wood Thrush & 0.0 & 0.0 & 0.0 & 0.0 \\
\hline \multicolumn{5}{|l|}{ Gray Catbird } \\
\hline \multicolumn{5}{|l|}{ Northern Mockingbird } \\
\hline Brown Thrasher & $\mathrm{v}$ & 3.3 & 0.0 & $\mathrm{v}$ \\
\hline \multicolumn{5}{|l|}{ Northern Parula } \\
\hline \multicolumn{5}{|l|}{ Yellow-throated Warbler } \\
\hline Pine Warbler & 32.8 & 29.5 & 19.7 & 16.4 \\
\hline Prairie Warbler & 31.2 & 9.8 & 18.0 & 1.6 \\
\hline \multicolumn{5}{|l|}{ Black-and-white Warbler } \\
\hline \multicolumn{5}{|l|}{ American Redstart } \\
\hline \multicolumn{5}{|l|}{ Prothonotary Warbler } \\
\hline \multicolumn{5}{|l|}{ Worm-eating Warbler } \\
\hline \multicolumn{5}{|l|}{ Swainson's Warbler } \\
\hline Ovenbird & $\mathrm{v}$ & 0.0 & 0.0 & 0.0 \\
\hline \multicolumn{5}{|l|}{ Louisiana Waterthrush } \\
\hline \multicolumn{5}{|l|}{ Kentucky Warbler } \\
\hline Common Yellowthroat & 1.6 & $\mathrm{v}$ & 0.0 & 0.0 \\
\hline \multicolumn{5}{|l|}{ Hooded Warbler } \\
\hline \multicolumn{5}{|l|}{ Yellow-breasted Chat } \\
\hline Summer Tanager & 8.2 & 8.2 & 3.3 & 3.3 \\
\hline Eastern Towhee & 14.8 & 8.2 & 16.4 & 4.9 \\
\hline Bachman's Sparrow & 21.3 & 16.4 & 9.8 & 9.8 \\
\hline Chipping Sparrow & 9.8 & 21.3 & 23.0 & 11.5 \\
\hline Field Sparrow & 0.0 & 0.0 & $\mathrm{~V}$ & 0.0 \\
\hline Northern Cardinal & $*$ & 1.6 & 6.6 & $\mathrm{v}$ \\
\hline Blue Grosbeak & 3.3 & 6.6 & $\mathrm{v}$ & 3.3 \\
\hline Indigo Bunting & 0.0 & 8.2 & $\mathrm{v}$ & 3.3 \\
\hline \multicolumn{5}{|l|}{ Eastern Meadowlark } \\
\hline Common Grackle & 0.0 & 0.0 & $\mathrm{v}$ & 0.0 \\
\hline Brown-headed Cowbird & $\mathrm{v}$ & $\mathrm{v}$ & $\mathrm{v}$ & 0.0 \\
\hline \multicolumn{5}{|l|}{ Orchard Oriole } \\
\hline American Goldfinch & 0.0 & 0.0 & 0.0 & $\mathrm{v}$ \\
\hline \multicolumn{5}{|l|}{ Visitors } \\
\hline American Robin & 0.0 & 0.0 & 0.0 & $\mathrm{v}$ \\
\hline total species & 26 & 29 & 22 & 20 \\
\hline
\end{tabular}


Scrub Oak Plot Census Data. Numbers are territories/40 ha.

$*=<0.5$ territory on plot, $\mathrm{v}=$ visitor

\begin{tabular}{|c|c|c|c|c|c|}
\hline Year & 1992 & 19931994 & 19951996 & 1997 & 1999 \\
\hline Observer & & JP & CI & MG & SW \\
\hline Plot area (ha) & & 12.2 & 12.2 & 12.2 & 12.2 \\
\hline Census Trips & & 11 & 10 & 10 & 10 \\
\hline \multicolumn{6}{|l|}{ Cooper's Hawk } \\
\hline Red-shouldered Hawk & & $\mathrm{v}$ & $\mathrm{v}$ & * & $\mathrm{v}$ \\
\hline Red-tailed Hawk & & $\mathrm{v}$ & 0.0 & 0.0 & $\mathrm{v}$ \\
\hline Wild Turkey & & $\mathrm{v}$ & 0.0 & 0.0 & 0.0 \\
\hline Northern Bobwhite & & 0.0 & 0.0 & 0.0 & 0.0 \\
\hline Mourning Dove & & 6.6 & 3.3 & $\mathrm{v}$ & 3.3 \\
\hline \multicolumn{6}{|l|}{ Common Ground-dove } \\
\hline Yellow-billed Cuckoo & & 6.6 & 3.3 & $\mathrm{v}$ & 3.3 \\
\hline \multicolumn{6}{|l|}{ Eastern Screech-Owl } \\
\hline Barred Owl & & 0.0 & 0.0 & 0.0 & $\mathrm{v}$ \\
\hline Common Nighthawk & & 0.0 & $\mathrm{v}$ & 0.0 & 0.0 \\
\hline \multicolumn{6}{|l|}{ Chuck-will's-widow } \\
\hline \multicolumn{6}{|l|}{ Whip-poor-will } \\
\hline \multicolumn{6}{|l|}{ Chimney Swift } \\
\hline \multicolumn{6}{|l|}{ Ruby-throated } \\
\hline Hummingbird & & $\mathrm{v}$ & 0.0 & 0.0 & 0.0 \\
\hline \multicolumn{6}{|l|}{ Red-headed Woodpecker } \\
\hline Red-bellied Woodpecker & & 3.3 & 3.3 & 0.0 & 0.0 \\
\hline Downy Woodpecker & & $*$ & $\mathrm{v}$ & 0.0 & 0.0 \\
\hline Northern Flicker & & $\mathrm{v}$ & $\mathrm{v}$ & 0.0 & $\mathrm{v}$ \\
\hline Pileated Woodpecker & & 4.9 & $\mathrm{v}$ & $\mathrm{v}$ & * \\
\hline Eastern Wood-Pewee & & 0.0 & 0.0 & $\mathrm{v}$ & 0.0 \\
\hline \multicolumn{6}{|l|}{ Acadian Flycatcher } \\
\hline \multicolumn{6}{|l|}{ Eastern Phoebe } \\
\hline Great Crested Flycatcher & & 11.5 & 6.6 & 3.3 & 4.9 \\
\hline \multicolumn{6}{|l|}{ Eastern Kingbird } \\
\hline \multicolumn{6}{|l|}{ White-eyed Vireo } \\
\hline \multicolumn{6}{|l|}{ Yellow-throated Vireo } \\
\hline Red-eyed Vireo & & $*$ & 0.0 & 0.0 & 0.0 \\
\hline Blue Jay & & 6.6 & 3.3 & $*$ & $\mathrm{v}$ \\
\hline American Crow & & $\mathrm{v}$ & $\mathrm{v}$ & 0.0 & $*$ \\
\hline Fish Crow & & 0.0 & $\mathrm{v}$ & 0.0 & 0.0 \\
\hline Purple Martin & & 0.0 & $\mathrm{v}$ & 0.0 & 0.0 \\
\hline Barn Swallow & & 0.0 & $\mathrm{v}$ & 0.0 & 0.0 \\
\hline Carolina Chickadee & & 4.9 & 3.3 & 6.6 & 8.2 \\
\hline Tufted Titmouse & & 6.6 & 3.3 & 3.3 & 6.6 \\
\hline \multicolumn{6}{|l|}{ White-breasted Nuthatch } \\
\hline Brown-headed Nuthatch & & $\mathrm{v}$ & 3.3 & * & $\mathrm{v}$ \\
\hline Carolina Wren & & 0.0 & $\mathrm{v}$ & $\mathrm{v}$ & 0.0 \\
\hline Blue-gray Gnatcatcher & & 4.9 & 3.3 & $\mathrm{v}$ & 18.0 \\
\hline Eastern Bluebird & & 0.0 & 0.0 & 0.0 & $\mathrm{v}$ \\
\hline
\end{tabular}


Scrub Oak Plot Census Data (cont.)

Wood Thrush

4.9

1.6

0.0

0.0

Gray Catbird

Northern Mockingbird

Brown Thrasher

$3.3 \quad 1.6$

1.6
0.0

0.0

0.0

$\mathrm{v}$

Northern Parula

0.0

6.6

Yellow-throated Warbler

18.0

6.6

3.3

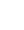

Prairie Warbler

0.0

$\mathrm{v}$

0.0

6.6

Black-and-white Warbler

3.3

3.3

0.0

American Redstart

Prothonotary Warbler

Worm-eating Warbler

Swainson's Warbler

Ovenbird

8.2

4.9

6.6

4.9

Louisiana Waterthrush

Kentucky Warbler

Common Yellowthroat

Hooded Warbler

Yellow-breasted Chat

Summer Tanager

11.5

6.6

3.3

4.9

Eastern Towhee

Bachman's Sparrow

Chipping Sparrow

0.0

$\mathrm{v}$

0.0

0.0

Field Sparrow

Northern Cardinal

3.3

v

4.9

Blue Grosbeak

Indigo Bunting

Eastern Meadowlark

Common Grackle

Brown-headed Cowbird

Orchard Oriole

$\begin{array}{cc}0.0 & 0.0 \\ \mathrm{~V} & \mathrm{v}\end{array}$

$\mathrm{v}$

0.0

0.0

0.0

$\mathrm{v}$

American Goldfinch

$\begin{array}{cc}\mathrm{v} & 0.0 \\ \mathrm{~V} & 0.0 \\ 0.0 & 0.0 \\ 0.0 & \mathrm{v}\end{array}$

Visitors

total species

18

15

9

13 
Tupelo-Cypress Swamp Plot Census Data. Numbers are territories/40 ha.

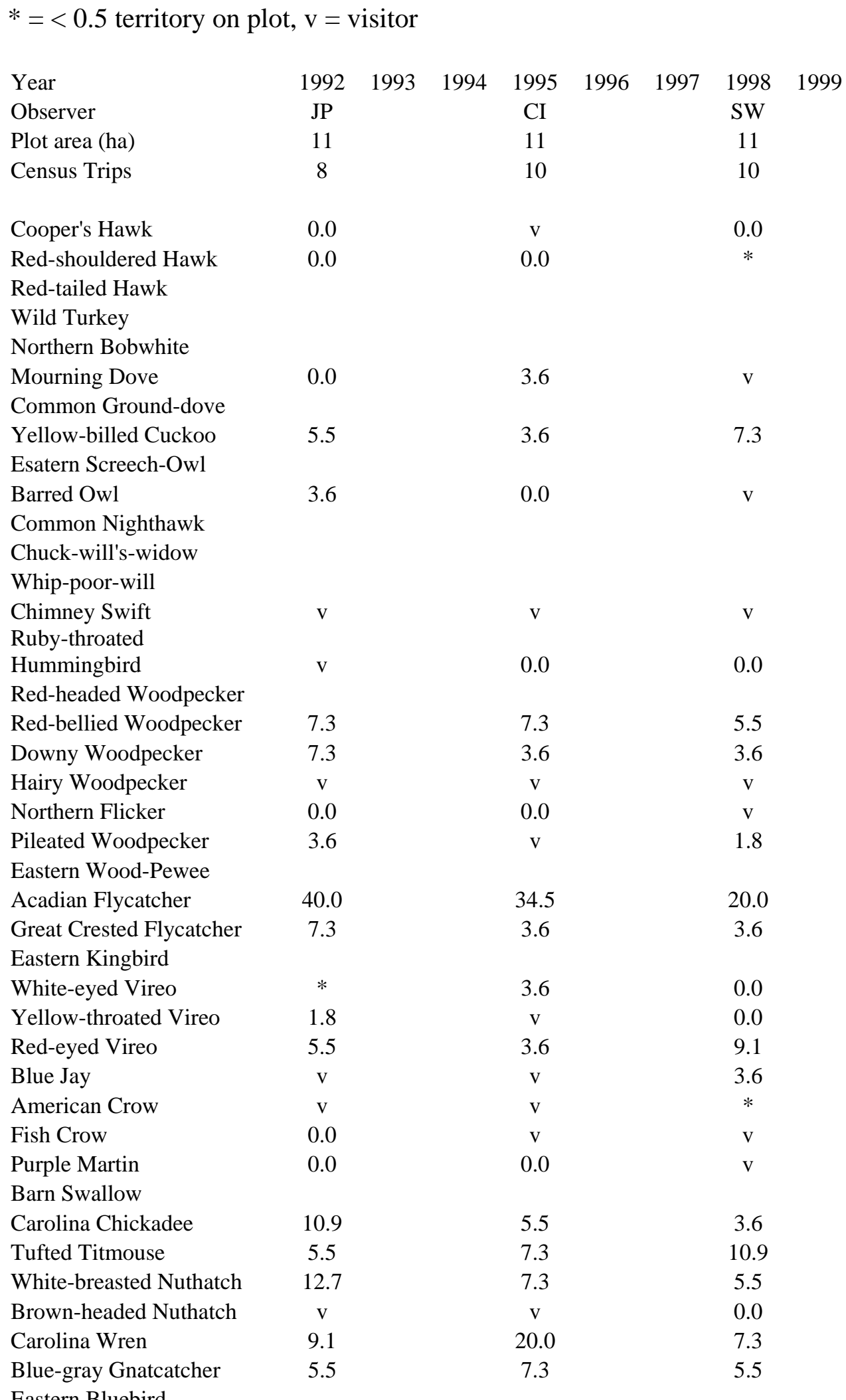

Eastern Bluebird 


\section{Tupelo-Cypress Swamp Plot Census Data (cont.)}

Wood Thrush

Gray Catbird

Northern Mockingbird

Brown Thrasher

Northern Parula

50.9

49.1

40.0

Yellow-throated Warbler

12.7

$\mathrm{V}$

0.0

Pine Warbler

Prairie Warbler

Black-and-white Warbler

American Redstart

1.8

0.0

0.0

Prothonotary Warbler

36.4

23.6

29.1

Worm-eating Warbler

Swainson's Warbler

Ovenbird

Louisiana Waterthrush

Kentucky Warbler

Common Yellowthroat

Hooded Warbler

Yellow-breasted Chat

Summer Tanager

Eastern Towhee

Bachman's Sparrow

Chipping Sparrow

Field Sparrow

Northern Cardinal

Blue Grosbeak

Indigo Bunting

Eastern Meadowlark

Common Grackle

Brown-headed Cowbird

$\mathrm{V}$

Orchard Oriole

American Goldfinch

\section{Visitors}

Anhinga

Great Blue Heron

Great Egret

White Ibis

Wood Stork

Wood Duck

total species

0.0

$*$

*

V

$\mathrm{V}$

$\mathrm{V}$

19
V

$\mathrm{V}$

0.0

$\mathrm{v}$

0.0

V

18 
Upland Hardwood Plot Census Data. Numbers are territories/40 ha.

$*=<0.5$ territory on plot, $\mathrm{v}=$ visitor

\begin{tabular}{|c|c|c|c|c|c|c|c|}
\hline Year & 1992 & 1993 & 1994 & 1995 & 1996 & 1997 & 1998 \\
\hline Observer & JP & & CI & & MG & & SW \\
\hline Plot area (ha) & 12.2 & & 12.2 & & 12.2 & & 12.2 \\
\hline Census Trips & 8 & & 10 & & 10 & & 10 \\
\hline
\end{tabular}

Cooper's Hawk

Red-shouldered Hawk

Red-tailed Hawk

Wild Turkey

Northern Bobwhite

Mourning Dove

Common Ground-dove

Yellow-billed Cuckoo

0.0

$\mathrm{V}$

$\mathrm{V}$

$\begin{array}{lllll}0.0 & 6.6 & 0.0 & \mathrm{v}\end{array}$

Eastern Screech-Owl

Barred Owl

4.9

6.6

4.9

6.6

Common Nighthawk

3.3

1.6

3.3

$\mathrm{v}$

Chuck-will's-widow

Whip-poor-will

0.0

Chimney Swift

Ruby-throated

Hummingbird

Red-headed Woodpecker

Red-bellied Woodpecker

Downy Woodpecker

Hairy Woodpecker

Northern Flicker

Pileated Woodpecker

Eastern Wood-Pewee

Acadian Flycatcher

Great Crested Flycatcher

Eastern Kingbird

White-eyed Vireo

Yellow-throated Vireo

Red-eyed Vireo

Blue Jay

American Crow

Fish Crow

Purple Martin

Barn Swallow

Carolina Chickadee

Tufted Titmouse

White-breasted Nuthatch

0.0

0.0

3.3

0.0

$\mathrm{v}$

0.0

3.3

3.3

0.0

0.0

1.6

0.0

18.0

0.0

0.0

$\mathrm{v}$

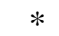

0.0

3.3

4.9

23.0

0.0

0.0

0.0

0.0

0.0

9.8

$\mathrm{v}$

0.0

0.0

$\mathrm{v}$

*

$\begin{array}{lll}4.9 & 6.6 & 3.3\end{array}$

0.0

$\begin{array}{cc}0.0 & \mathrm{v} \\ 4.9 & 0.0\end{array}$

$\begin{array}{ccc}0.0 & 4.9 & 0.0 \\ \mathrm{v} & 3.3 & 3.3\end{array}$

$\mathrm{v}$

$0.0 \quad 0.0$

V

29.5

9.8

0.0

6.6

$\mathrm{V}$

$\begin{array}{lll}0.0 & 0.0 & 0.0\end{array}$

1.6

$\mathrm{V}$

0.0

11.5

8.2

3.3

13.1

0.0

0.0

0.0

0.0

26.2

13.1

$\mathrm{v}$

0.0

0.0

0.0

13.1

6.6

11.5

3.3

8.2

6.6

13.1

3.3

9.8

1.6

0.0

$\mathrm{v}$

Brown-headed Nuthatch

Carolina Wren

16.4

11.5

13.1

8.2

Blue-gray Gnatcatcher

4.9

9.8

9.8

8.2 
Upland Hardwood Plot Census Data (cont.)

\begin{tabular}{|c|c|c|c|c|}
\hline $\begin{array}{l}\text { Wood Thrush } \\
\text { Gray Catbird } \\
\text { Northern Mockingbird } \\
\text { Brown Thrasher }\end{array}$ & 16.4 & 13.1 & 8.2 & 6.6 \\
\hline Northern Parula & 39.4 & 34.4 & 72.2 & 34.4 \\
\hline Yellow-throated Warbler & 3.3 & 11.5 & 23.0 & 3.3 \\
\hline Pine Warbler & 1.6 & $\mathrm{v}$ & 0.0 & $\mathrm{v}$ \\
\hline \multicolumn{5}{|l|}{ Prairie Warbler } \\
\hline Black-and-white Warbler & 4.9 & 4.9 & 6.6 & $\mathrm{v}$ \\
\hline American Redstart & 3.3 & $\mathrm{v}$ & 6.6 & 0.0 \\
\hline Prothonotary Warbler & $*$ & 1.6 & $\mathrm{v}$ & $\mathrm{v}$ \\
\hline Worm-eating Warbler & $\mathrm{v}$ & 0.0 & 6.6 & $\mathrm{v}$ \\
\hline \multicolumn{5}{|l|}{ Swainson's Warbler } \\
\hline Ovenbird & $\mathrm{v}$ & $\mathrm{v}$ & 0.0 & 0.0 \\
\hline Louisiana Waterthrush & $\mathrm{v}$ & 3.3 & 6.6 & $*$ \\
\hline Kentucky Warbler & 16.4 & 4.9 & $\mathrm{v}$ & 0.0 \\
\hline Common Yellowthroat & 0.0 & 0.0 & $\mathrm{v}$ & 0.0 \\
\hline Hooded Warbler & 16.4 & 9.8 & 6.6 & 1.6 \\
\hline \multicolumn{5}{|l|}{ Yellow-breasted Chat } \\
\hline Summer Tanager & 13.1 & 11.5 & 9.8 & 8.2 \\
\hline \multicolumn{5}{|l|}{ Eastern Towhee } \\
\hline \multicolumn{5}{|l|}{ Bachman's Sparrow } \\
\hline \multicolumn{5}{|l|}{ Chipping Sparrow } \\
\hline \multicolumn{5}{|l|}{ Field Sparrow } \\
\hline Northern Cardinal & 11.5 & 8.2 & 11.5 & 0.0 \\
\hline Blue Grosbeak & 0.0 & $\mathrm{v}$ & 0.0 & $\mathrm{v}$ \\
\hline Indigo Bunting & 0.0 & 0.0 & $\mathrm{v}$ & $\mathrm{v}$ \\
\hline \multicolumn{5}{|l|}{ Eastern Meadowlark } \\
\hline \multicolumn{5}{|l|}{ Common Grackle } \\
\hline Brown-headed Cowbird & 0.0 & $\mathrm{v}$ & $\mathrm{v}$ & $\mathrm{v}$ \\
\hline Orchard Oriole & & & & \\
\hline American Goldfinch & 0.0 & 0.0 & 0.0 & $\mathrm{v}$ \\
\hline
\end{tabular}

\section{Visitors}

